\title{
Haldane-Gapped Spin Chains as Luttinger Liquids: Correlation Functions at Finite Field
}

\author{
Robert M. Konik and Paul Fendley \\ Department of Physics \\ University of Virginia \\ Charlottesville, VA 22904-4714
}

June 2, 2001

\begin{abstract}
We study the behavior of Heisenberg, antiferromagnetic, integer-spin chains in the presence of a magnetic field exceeding the attendant spin gap. For temperatures much smaller than the gap, the spin chains exhibit Luttinger liquid behavior. We compute exactly both the corresponding Luttinger parameter and the Fermi velocity as a function of magnetic field. This enables the computation of a number of correlators from which we derive the spin conductance, the expected form of the dynamic structure factor relevant to inelastic neutron scattering experiments, and NMR relaxation rates. We also comment upon the robustness of the magnetically induced gapless phase both to finite temperature and finite couplings between neighbouring chains.
\end{abstract}

\section{Introduction}

The existence of a gap in one-dimensional, integer-spin, Heisenberg antiferromagnets was first predicted by Haldane [1]. He found that such spin chains can be mapped onto a gapped field theory in the large-spin, continuum limit. A variety of checks imply that this behavior persists down to spin $s=1$. A spin-1 chain with a specific $(\vec{S} \cdot \vec{S})^{2}$ coupling has been rigorously shown to exhibit a spin gap 4 . While at a differing value of the $(\vec{S} \cdot \vec{S})^{2}$ coupling, the spin chain is gapless [5], this critical point is believed to be unstable in the two-parameter space of couplings. Gapless behavior thus only arises as a product of fine-tuning. Numerous numerical studies carried out on spin-1 chains observe a gap [6]. Experimentally, inelastic neutron scattering studies on a number of quasi-one-dimensional spin-1-chain materials are consistent with a finite spin gap [7, 8, 9].

The physics underlying the gap is particularly robust: related systems such as two-leg spin$1 / 2$ or Hubbard ladders also exhibit a gap to spin excitations [10. Roughly speaking, integer-spin composites form across the rungs of the ladder making it into an effective integer-spin chain. Both the ability to fabricate these materials and their relationship to high $T_{c}$ cuprate superconductors have made them the focus of intense theoretical and experimental study [1, 12. 
The centrality of the gap is no less when these materials are subjected to an applied Zeeman field, $H$. In a field exceeding some critical value, $H_{c} \sim \Delta$, the excitation spectrum of integerspin chains changes dramatically and the gap vanishes [13, 14]. At $H=0$ the ground state is a singlet. The elementary excitations above the ground state are three massive spin-one bosonic magnon modes. In contrast, when $H$ exceeds $H_{c}$, the gap of one of the magnons closes and the ground state of the spin chain begins to fill in with gapless excitations. If interactions between the gapless magnons were completely absent, the excitations would collapse into a condensate of free bosons. On the other hand if interactions were perfectly repulsive at any energy scale, the bosons could be thought to possess a hard core and so form a gas of free fermions with some Fermi surface. But in fact the interactions are expected to lie midway between these extremes and what one ends up with is equivalent to an interacting sea of fermions. This of course is nothing more than a Luttinger liquid. The purpose of this article is to study its properties.

The field theory describing the continuum limit of integer-spin chains is the $O(3)$ non-linear sigma model (NLSM) without topological term [1, 2]. The $O(3)$ NLSM also describes halfinteger-spin chains but in this case the topological term is present with coupling $\theta=\pi$. The presence of the topological term leads to gapless behavior. Indeed at low energies the $O(3)$ NLSM with $\theta=\pi$ flows to the critical theory, $S U(2)_{1}$ (for a review see [2]).

Unlike its ancestral theory, the Heisenberg spin-1 chain, the $O(3)$ sigma model has the virtue of being integrable [3, 15. While aspects of the physics of integer-spin chains have been studied using the integrability of the $O(3) \operatorname{NLSM}[14,16,17$, 18, spin chains remain incompletely understood. While thermodynamic properties of an integrable model are generically accessible, correlation functions are not. There do exist a variety of techniques to compute correlators. At zero temperature, truncated 'form-factor' computations are able to access exact information of the low-energy properties of the spectral functions [12, 16]. These techniques have recently been extended to compute exact low temperature expansions of these same quantities [19]. There also exists an elegant semi-classical approach [20, 21] predicated upon combining ultra-low-energy information from the quantum theory with older approaches of computing correlators in classical systems [22]. However, all of these techniques require the energy scales in the problem (in particular, the temperature, $T$, and the applied field, $H$ ) to be much smaller than the gap, $\Delta$.

Fortuitously there exists an alternate approach which allows the computation of correlation functions when $H>H_{c}$. The control over the thermodynamic properties of the model that integrability affords allows the computation of the Luttinger parameter. While the Luttinger parameter characterizes the strength of the interactions between excitations, it is a far more central quantity in that it provides a near complete description of the low-energy structure of the theory. Together with the knowledge of the Fermi velocity, a quantity also easily accessible with integrability, a host of information can be determined, including the computation of specific correlators and their scaling exponents. Such techniques have been used to study the sine-Gordon model in the presence of a chemical potential exceeding the gap (i.e. the mass of the sine-Gordon solitons) [23, 24]. Similarly the low-energy structure of doped generic Hubbard ladders/armchair carbon nanotubes has been studied in [12] utilizing this formalism. The particular derivation of the Luttinger parameter in this paper is based upon the treatment in [25].

The ability to compute the Luttinger parameter together with the Fermi velocity is predicated upon some generic properties of integrable models. Most importantly, the exact eigenfunctions of the model's fully interacting Hamiltonian are known. With this knowledge comes a well- 
defined notion of 'particles' or elementary excitations in the system. Ultimately this feature is a consequence of the infinite number of conservation laws possessed by the integrable model. In particular, particle number is conserved in any collision and multi-particle $S$ matrix elements factorize into products of two-particles ones. An integrable model is a superior version of a Fermi liquid: a particle's lifetime is infinite regardless of distance from the Fermi surface.

In order to appreciate these features of the $O(3)$ NLSM, we provide an overview of the model. The model is described by the action,

$$
S=\frac{1}{2 g} \int d x d t\left(\partial^{\mu} \mathbf{n} \partial_{\mu} \mathbf{n}\right)
$$

where $\mathbf{n}=\left(n_{x}, n_{y}, n_{z}\right)$ is a bosonic vector field constrained to live on the unit sphere, $\mathbf{n} \cdot \mathbf{n}=1$. This action is arrived at from the Hamiltonian of the spin chain,

$$
H=J \sum_{i} S_{i} \cdot S_{i+1}
$$

In the continuum, large- $s$, limit, the spin operator, $S_{i}$, is related to the field, $\mathbf{n}$, via

$$
S_{i}=(-1)^{i} s n_{i}+M_{i}
$$

$\mathbf{n}(x, t)$ is the sub-lattice or Néel order parameter while $\mathbf{M}$ describes the uniform (i.e. wavevector $k \sim 0)$ magnetization. $\mathbf{M}$ is related to $\mathbf{n}$ via

$$
\mathbf{M}=\frac{1}{g} \mathbf{n} \times \partial_{t} \mathbf{n},
$$

and so is given in terms of the momentum conjugate to $\mathbf{n}$.

The triplet of bosons which form the low-energy excitations of the $O(3)$ NLSM have a relativistic dispersion relation given by

$$
E(p)=\left(p^{2}+\Delta^{2}\right)^{1 / 2}
$$

Here $\Delta$ is the energy gap or mass of the bosons and is related to the bare coupling, $g$, via $\Delta \sim J e^{-\pi / g}$. We have set the bare spin-wave velocity, $v_{s}=2 J s$ (the speed of light in this relativistic system) to be 1 . The dispersion relation of all three bosons is identical as the model has a global $\mathrm{O}(3)$ symmetry. We stress that this relativistic invariance is a natural feature of the low energy structure of the spin chain.

Our approach to the Luttinger liquid phase of the Heisenberg spin-1 chain shares some similarities with others taken in the literature. The NLSM has been used previously to study the field-induced gapless phase of spin-1 chains, albeit in the context of large- $N$ techniques 28], where the $O(3)$ NLSM is replaced with its $O(N)$ counterpart. (Parenthetically, we point out that the large N approximation of the $O(3)$ NLSM have been criticized by [29]. In this work the authors note that the ultra low energy limit of the scattering in the $O(3)$ NLSM differs from that predicted by a large $\mathrm{N}$ expansion of the exact $O(N)$ S-matrix.)

Beyond large- $N$, the Luttinger liquid phase of the spin-1 chain has also been studied in the guise of analyzing spin-1/2 ladders. Bosonization techniques have been used to study the ladder system in a regime where the legs of the ladders are weakly coupled 33 . In the opposite 
limit, in a regime where there are strong antiferromagnetic correlations across the ladder's rungs, the gapless Luttinger liquid behaviour has been explored through mapping the system onto an effective XXZ spin-1/2 chain [30. In this latter case, the underlying integrability of the XXZ spin chain can be brought to bear upon the problem [31. Given the simplicity of the map onto the spin-1/2 chain, we adapt the map to the case where there are ferromagnetic interactions across the rungs of the ladder (appropriate for describing a spin-1 chain). Our primary purpose in doing so is to come up with a more precise identification between the relevant fields in the theory and the bosonic degrees of freedom of the Luttinger liquid. Although we could exploit the integrability of the spin-1/2 chain to compute the Luttinger parameter, we do not do so. Given that the map to the effective spin- $1 / 2$ chain is done through a perturbative expansion where the importance of the higher order terms in the series is uncertain, we view extracting the Luttinger parameter directly from the $O(3)$ NLSM as more reliable.

Haldane gap spin chains in a magnetic field have also been studied through a mapping onto an interacting, spinless Bose gas [34. This map provides a reliable description of the spin chain at small magnetizations. At small magnetizations, the low density of excitations forming the ground state interact only weakly. The scaling exponents and thermodynamic properties of the system are then independent of the exact nature of these interactions. Although not done by the authors of [34, the integrability of the Bose gas, like the integrability of the $O(3)$ NLSM, could be used to determine the various properties of the Luttinger liquid phase. However beyond the low magnetization regime, the results would differ. It is interesting to note, however, that by fine tuning the strength of the interactions of the Bose gas, the analysis of the $O(3)$ NLSM, in particular the computation of the Luttinger parameter, can be reproduced in large degree. We comment upon this further in Section 3.

In this article we take the Hamiltonian of the spin chain to be in its minimal Heisenberg form and so ignore (for the most part) the affects of anisotropies upon the physics. These can take (at least) two forms. Easy-axis anisotropies,

$$
\Delta H=D_{x} \sum_{i}\left(S_{x i}\right)^{2}+D_{y} \sum_{i}\left(S_{y i}\right)^{2}+D_{z} \sum_{i}\left(S_{z i}\right)^{2},
$$

of varying strengths are often found in spin-1 chain materials. Additionally, actual spin chain materials never take the form of an isolated chain. Rather the chains exist in three dimensional arrays with weak but non-zero interchain couplings, $J^{\prime}$. Thus the chains are at best quasi-onedimensional. With a finite $J^{\prime}$, there will be some correspondingly finite Néel temperature, $T_{N}$. Below $T_{N}$ the physics will be dramatically different than described in this article.

$\mathrm{CsNiCl}_{3}$ was the first material for which evidence of a Haldane gap was found [8. This material suffers from the second aforementioned anisotropy with a relatively large interchain coupling, $J^{\prime} / J \sim .017$. Consequently Néel order was observed to set in at $T \sim 5 K$. A more promising material for the observation of a Haldane gap was found in $\mathrm{Ni}\left(\mathrm{C}_{2} \mathrm{H}_{8} \mathrm{~N}_{2}\right)_{2} \mathrm{NO}_{2} \mathrm{ClO}_{4}$ (NENP). For NENP, the ratio $J^{\prime} / J \sim 6 \times 10^{-4}$ is sufficiently small that 3D Néel order has not been observed down to temperatures $\sim 1.2 \mathrm{~K}[9]$. However this material is characterized by a large easy-axis anisotropy, $D_{z} / J \sim .25 ; D_{z} / \Delta \sim 5 / 8\left(D_{x} \sim D_{y} \sim 0\right)$. Related materials $\mathrm{Ni}\left(\mathrm{C}_{5} \mathrm{H}_{14} \mathrm{~N}_{2}\right)_{3}\left(\mathrm{PF}_{6}\right)$ (NDMAP) and $\mathrm{Ni}\left(\mathrm{C}_{5} \mathrm{H}_{14} \mathrm{~N}_{2}\right)_{2} \mathrm{~N}_{3}\left(\mathrm{ClO}_{4}\right)$ (NDMAZ) share similar easy-axis anisotropies. In terms of our analysis, these latter compounds share the additional unwanted feature of field-induced antiferromagnetism [36. The Luttinger liquid that results from magnetic fields large enough to extinguish the Haldane gap leads to quasi-long range antiferromagnetic 
correlations. With a small finite $J^{\prime}$, these quasi-long range correlations are promoted to full fledged long range order. The corresponding Néel temperature increases with applied magnetic fields. In a mean field framework, we compute this ordering temperature in Section 4. Thus at fixed temperature we expect only a finite window in the applied magnetic field in which the Luttinger liquid behavior will persist.

Perhaps the material best suited to the analysis presented in this paper is $A g V P_{2} S_{6}$. It has an extremely small interchain coupling, $J^{\prime} / J \sim 10^{-5}$ and a similarly small easy axis anisotropy, $D_{z} / \Delta \sim 10^{-2}$. However it possesses a comparatively large gap, $\Delta \sim 320 K$. As such, high field measurements $(H>\Delta)$ have yet to be done on this material (and are unlikely to be done soon), opposite to the case of NENP [37], with a much smaller average gap, $\Delta \sim 20 K$.

The paper is organized as follows. In Section 2 we develop a Landau-Ginzburg description of the low-energy effective theory of the integer-spin chain in a magnetic field exceeding the spin gap. This effective theory reduces to a Luttinger liquid and so has two controlling parameters: the Luttinger parameter, $K$, and the Fermi velocity, $v_{F}$. In Section 3 we show how these parameters can be determined as a function of the applied field, $H$, through consistency with the $O(3)$ NLSM.

With this description of the low-energy theory in hand, we analyze the behavior of a number of correlators in Section 4. Using a Kubo formula together with our knowledge of the currentcurrent correlators, we compute the spin conductance and the static susceptibility. With this latter quantity we have thus come full circle. We computed $K$ and $v_{F}$ based upon thermodynamic considerations and then in turn computed correlators. From these same correlators we then (re)compute (consistently) thermodynamic quantities. We also study the staggered spin-spin correlators, quantities which would be probed both in inelastic neutron scattering experiments near wavevector $k=\pi$ and NMR relaxation rate measurements.

While we have already raised the issue of the effects of interchain couplings upon the stability of the Luttinger liquid phase, we also consider in Section 4 the robustness of this phase to finite temperature. Although our derivation of the effective low-energy theory is done at zero temperature, the conformal/scale invariant nature of the theory allows us to easily determine quantities at $T>0$. To explore how large the temperature relative to the gap may become before the Luttinger picture breaks down, we study the susceptibility at finite $T$ and $H$. We do so using a more sophisticated description of the system, the exact equations (good for arbitrary $T$ and $H$ ) giving the system's free energy.

\section{Low-Energy Effective Theory for $H>\Delta$}

In this section we describe the emergent Luttinger liquid behavior of a spin chain arising in magnetic fields larger than the gap and temperatures satisfying $T \ll \Delta$. Following [26], we provide a corresponding Landau-Ginzburg description. Although we work with an effective theory, we will be able to compute the various phenomenological parameters appearing in it by insisting on consistency with the $O(3)$ NLSM. This will form the topic of Section 3.

The Landau-Ginzburg description is an approximate field theory of the magnons and their interactions. We represent the magnon field as $\mathbf{m}$. It is a vector under the $O(3)$ symmetry akin to original field $\mathbf{n}$ and shares all of $\mathbf{n}$ 's original discrete symmetries. However $\mathbf{m}$ is not constrained to live on the unit sphere. The magnons have a gap and a relativistic dispersion 
relation. The simplest effective Hamiltonian with these characteristics is [26],

$$
\mathcal{H}=\frac{1}{2}\left(\boldsymbol{\Pi}^{2}+\left(\partial_{x} \mathbf{m}\right)^{2}\right)+\frac{\Delta^{2}}{2}|\mathbf{m}|^{2}+\lambda|\mathbf{m}|^{4}-H \cdot(\mathbf{m} \times \mathbf{\Pi}),
$$

where $\boldsymbol{\Pi}$ is the momenta conjugate to $\mathbf{m}$. We have added a $|\mathbf{m}|^{4}$ term to ensure overall stability. The corresponding Lagrangian (with $H$ in the $z$ direction) is

$$
\mathcal{L}=\frac{1}{2}\left(\left(\partial_{t} \mathbf{m}\right)^{2}-\left(\partial_{x} \mathbf{m}\right)^{2}\right)+H\left(m_{x} \partial_{t} m_{y}-m_{y} \partial_{t} m_{x}\right)+\frac{H^{2}}{2}\left(m_{x}^{2}+m_{y}^{2}\right)-\frac{\Delta^{2}}{2}|\mathbf{m}|^{2}-\lambda|\mathbf{m}|^{4} \cdot(8)
$$

The last three terms form the effective potential for the model.

When $H<\Delta$, the minimum of the Landau-Ginzburg potential occurs for $\mathbf{m}=0$. But when $H>\Delta$ the minima of the potential now occur for field configurations of the form

$$
\begin{aligned}
m_{x}^{2}+m_{y}^{2} & =\frac{H^{2}-\Delta^{2}}{4 \lambda} \\
m_{z} & =0 .
\end{aligned}
$$

As we are interested in the low-energy behavior of the theory, we focus upon the low-energy fluctuations about these field configurations. Introducing the new fields $m$ and $\Phi$ via

$$
\left(m+m_{o}\right) e^{ \pm i \Phi}=\frac{m_{x} \pm i m_{y}}{\sqrt{2}},
$$

with $m_{o}^{2}=\left(H^{2}-\Delta^{2}\right) /(8 \lambda)$, the effective Lagrangian can be rewritten (to quadratic order in the fields)

$$
\begin{gathered}
\mathcal{L}=\frac{1}{2}\left(\left(\partial_{t} m_{z}\right)^{2}-\left(\partial_{x} m_{z}\right)^{2}\right)+\left(\left(\partial_{t} m\right)^{2}+m_{o}^{2}\left(\partial_{t} \Phi\right)^{2}\right)-\left(\left(\partial_{x} m\right)^{2}+m_{o}^{2}\left(\partial_{x} \Phi\right)^{2}\right) \\
+2 H\left(m_{o}^{2}+2 m_{o} m\right) \partial_{t} \Phi-2\left(H^{2}-\Delta^{2}\right) m^{2}-\frac{H^{2}}{2} m_{z}^{2} .
\end{gathered}
$$

The low-energy physics in $\mathcal{L}$ is governed solely by the field $\Phi$. The $m$ and $m_{z}$ modes are massive and may be integrated out. Doing so results in a Lagrangian of the form

$$
\mathcal{L}=\frac{v_{F} K}{2 \pi}\left(\left(\partial_{x} \Phi\right)^{2}-\frac{1}{v_{F}^{2}}\left(\partial_{t} \Phi\right)^{2}\right)
$$

the bosonic form of the Luttinger model. Here $K$ and $v_{F}$ are the effective Luttinger parameter and Fermi velocity. These parameters can be related to the various parameters appearing in the original Landau-Ginzburg Hamiltonian. However this can be done only at the mean-field level; quantum fluctuations strongly renormalize their values. The approach we thus take is to access $K$ and $v_{F}$ directly from the integrability of the $O(3)$ NLSM. This, as has been indicated, is done in Section 3.

To complete this section we identify the fields of the $O(3)$ NLSM in terms of $\Phi$. We can factorize the boson, $\Phi$, into right and left moving pieces each describing the excitations at the two Fermi points, $\Phi=\phi_{R}+\phi_{L}$. The (Euclidean) propagators of $\phi_{L / R}$ are given in terms of $K$. We have

$$
\left\langle\phi_{L}(z) \phi_{L}(w)\right\rangle=-\frac{1}{4 K} \log (z-w)
$$




$$
\left\langle\phi_{R}(\bar{z}) \phi_{R}(\bar{w})\right\rangle=-\frac{1}{4 K} \log (\bar{z}-\bar{w}),
$$

where $z / \bar{z}=v_{F} \tau \pm i x$. The fields, $n_{ \pm}(x, \tau)$, creating $S_{z}=1$ excitations are given in terms of the boson, $\Phi$, by

$$
n^{ \pm}=e^{ \pm i \Phi},
$$

and so are governed by the propagator of the form

$$
\left\langle n_{+}(z) n_{-}(w)\right\rangle=\left(x^{2}+v_{F}^{2} \tau^{2}\right)^{-\frac{1}{4 K}} .
$$

We thus understand the spectral functions of low-energy excitations in terms of $K$ when $H>\Delta$. Moreover we can express the z-component of the magnetization operators in terms of the $U(1)$ boson currents (in Euclidean space). Fluctuations in the magnetization density are given by

$$
M_{0}^{3} \equiv M_{z}=-\frac{K}{v_{F} \pi} \partial_{\tau} \Phi=\frac{K}{\pi}\left(\partial_{\bar{z}} \phi_{R}+\partial_{z} \phi_{L}\right) \equiv \frac{1}{2 \pi}\left(j_{R}+j_{L}\right)
$$

while the corresponding spin current, $j_{S}$, equals

$$
M_{1}^{3} \equiv \frac{j_{S}}{v_{F}}=-\frac{K}{\pi} \partial_{x} \Phi=-\frac{i K}{\pi}\left(\partial_{\bar{z}} \phi_{R}-\partial_{z} \phi_{L}\right) \equiv \frac{i}{2 \pi}\left(j_{R}-j_{L}\right) .
$$

Current-current correlators then can be deduced from

$$
\left\langle j_{L}(z) j_{L}(w)\right\rangle=-\frac{K}{(z-w)^{2}},
$$

an immediate consequence of (13) .

The above identification of the fields is minimal in nature. It is based upon both the LandauGinsburg analysis together with the simplest association of the fields in the NLSM with those of the Luttinger liquid (12). However it misses subleading terms. To identify such terms directly from the Landau-Ginsburg analysis would seem difficult. To circumvent this difficulty, we analyze a spin-1/2 ladder system. With ferromagnetic interactions across an individual rung of the ladder we obtain an effective spin 1. And with antiferromagnetic interactions along the length of the ladder, we obtain an effective antiferromagnetic Heisenberg spin-1 chain. In a magnetic field, the ladder system can be reduced to an equivalent spin-1/2 chain. Bosonizing such a chain in the standard fashion yields a Luttinger liquid, precisely as in (12). Proceeding in this fashion offers us the advantage that it allows a more complete identification of the fields, including subleading terms. We so find

$$
\begin{aligned}
& M_{z}(x, \tau)=-\frac{K}{v_{F} \pi} \partial_{\tau} \Phi(x, \tau)+c \cos (2 \pi(\Theta(x, \tau)-M x)) \\
& n^{-}(x, \tau)=e^{-i \Phi(x, \tau)}\left(1+c_{1} \cos (2 \pi(\Theta(x, \tau)-M x)) .\right.
\end{aligned}
$$

Here $\Theta(x, \tau)$ is the boson dual to that of $\Phi$ and may be written as

$$
\Theta(x, \tau)=\frac{K}{\pi}\left(\phi_{L}(z)-\phi_{R}(\bar{z})\right)
$$


while $c$ and $c_{1}$ are some numerical constants. We see that the leading terms agree with that of the Landau-Ginsburg analysis. The subleading terms are interesting in that they depend upon the magnetization, $M$, of the system, and so lead to incommensurate behaviour. This incommensurate behaviour has been argued to occur [33] in the context of spin-1/2 ladders with ferromagnetic rungs.

The map from spin- $1 / 2$ ladders to effective spin- $1 / 2$ chains has been studied extensively. Two general approaches have been employed. In the case where the spin- $1 / 2$ ladder is strongly antiferromagnetically coupled across the rung (we are however interested in ferromagnetic couplings), a straightforward map takes the ladder in a magnetic field to a spin- $1 / 2$ chain [30, 31. In the case where the spin- $1 / 2$ ladder is weakly coupled across the rungs (either ferromagnetically or antiferromagnetically) more detailed studies have been carried out, using successive bosonizations/fermionizations [33]. Due to the simplicity of the former, we adapt this method to ferromagnetic rung couplings, as discussed in Appendix C.

The need to adapt the map is predicated upon two pieces of physics. The Luttinger parameter of the magnetically induced gapless phase of spin- $1 / 2$ ladders with ferromagnetic/antiferromagnetic rungs satisfies $K>1 / K<1$. However a naïve application of the map yields $K<1$ regardless of the type of rung coupling. Additionally, the map as it stands does not take into account the different types of gapless behaviour. The perpendicular susceptibility of ladders with antiferromagnetic rungs, for example, sees power law like decay at $k=(\pi, \pi)$ and exponential decay at $k=(\pi, 0)$. But the situation is reversed for ladders with ferromagnetic rungs.

\section{Computing $K$ and $v_{F}$ from the Integrability of the $O(3)$ NLSM}

In this section we present exact results for the $H>H_{c}$ phase of the $O(3)$ NLSM. They confirm the Luttinger behavior implied by the Landau-Ginzburg description discussed in the last section. In particular we show how to extract the phenomenological parameters, $K$ and $v_{F}$, appearing in this description from the integrability of the $O(3)$ NLSM.

We begin by describing how the ground state of the $O(3)$ NLSM is altered in the presence of a magnetic field. Neglecting interactions, the energy of an excitation with bare energy, $E$, in a finite magnetic field, $H$, along the $z$ axis is

$$
E-S_{z} H
$$

This leads to a splitting of the spin-1 triplet of magnons. In a mean-field 'semi-conductor' picture, this splitting is sketched in Figure 1. As the magnetic field, $H$, is increased beyond the critical value of $H=H_{c}=\Delta$, the ground state of the spin chain dramatically changes. The ground state now begins to fill in with a finite density of magnons (and so is akin to a doped semiconductor). If the $S_{z}=1$ magnons were non-interacting, they would condense in the lowest possible energy level. But the magnons interact repulsively, and so they fill the ground state as if they were fermions or hard-core bosons. The technical manifestation of this behavior is the minus sign in the $S$-matrix at zero momentum transfer $(\theta=0$ in (20) $)$. With $H$ larger than $\Delta$, the energy of an excitation is potentially negative and excitations carrying $S_{z}=1$ appear in the ground state. We illustrate this in Figure 1 schematically by plotting the dispersion relations for the three types of excitations in the system: $S_{z}=-1,0,1$. 

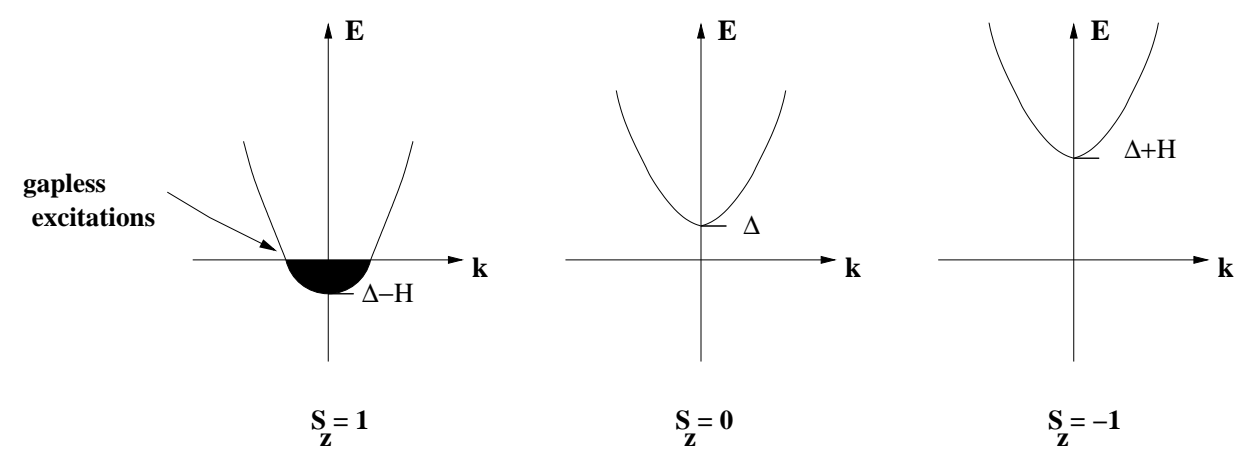

Figure 1: A sketch of the ground state of the $O(3)$ non-linear sigma model in the presence of a magnetic field that exceeds the gap, $\Delta$. The $S_{z}=1$ band is now partially filled with excitations; the $S_{z}=0$ band is unchanged as it does not couple to the magnetic field; and the $S_{z}=-1$ band is shifted upwards in energy.

The ground state of the system for $H>H_{c}$ is therefore a sea of magnons. The low-energy spin excitations consist of slight deformations of the filled sea in the $S_{z}=1$ band. These low-lying excitations can be of arbitrarily small energy and so are gapless. If we linearize such excitations above the Fermi energy of this band, we obtain a Luttinger liquid characterized by a Luttinger parameter, $K$, and a Fermi velocity, $v_{F}$.

To describe the ground state exactly we need to take into account interactions of the particles. These interactions are parameterized by their scattering. As the $O(3)$ sigma model is integrable, the scattering matrix is known exactly. Exploiting the theory's relativistic invariance, we can parameterize a particle's energy and momentum in terms of its rapidity $\theta$, defined as

$$
E=\Delta \cosh (\theta) ; \quad P=\Delta \sinh (\theta) .
$$

In this parameterization, Lorentz boosts send $\theta \rightarrow \theta+\alpha$. Lorentz invariant quantities like $S$ matrix elements therefore depend only on differences of rapidities. The $S$ matrix for scattering the two-particle state $\left|a_{1}\left(\theta_{1}\right) a_{2}\left(\theta_{2}\right)\right\rangle\left(a_{1}, a_{2}=1,2,3\right)$ into the final state $\left|a_{4}\left(\theta_{2}\right) a_{3}\left(\theta_{1}\right)\right\rangle$ is 3 ]

$$
S_{a_{1} a_{2}}^{a_{3} a_{4}}\left(\theta_{12}\right)=\delta_{a_{1} a_{2}} \delta_{a_{3} a_{4}} \sigma_{1}\left(\theta_{12}\right)+\delta_{a_{1} a_{3}} \delta_{a_{2} a_{4}} \sigma_{2}\left(\theta_{12}\right)+\delta_{a_{1} a_{4}} \delta_{a_{2} a_{3}} \sigma_{3}\left(\theta_{12}\right)
$$

where $\theta=\theta_{1}-\theta_{2}$ and

$$
\begin{aligned}
\sigma_{1}(\theta) & =\frac{2 \pi i \theta}{(\theta+i \pi)(\theta-i 2 \pi)} \\
\sigma_{2}(\theta) & =\frac{\theta(\theta-i \pi)}{(\theta+i \pi)(\theta-i 2 \pi)} \\
\sigma_{3}(\theta) & =\frac{2 \pi i(i \pi-\theta)}{(\theta+i \pi)(\theta-i 2 \pi)}
\end{aligned}
$$

Since the ground state is filled with solely $S_{z}=1$ particles, we need the $S$ matrix element, $S_{++}$, for scattering of two such particles:

$$
S_{++}(\theta)=\frac{\theta-i \pi}{\theta+i \pi}
$$


This is found from the change of basis, $A_{ \pm}=\frac{1}{\sqrt{2}}\left(A_{1}+i A_{2}\right)$, where here $A_{i}$ is an operator creating an excitation carrying quantum number $i$.

Knowing $S$ allows us to determine the density of states per unit length, $\rho(\theta)$, of the sea of particles at $H>H_{c}$. The derivation is standard and can be found for the case at hand in 38 . For completeness we repeat these arguments in Appendix A. At zero temperature, the repulsive nature of the particles leads them to fill the sea up to some Fermi momentum, so that the density satisfies $\rho(\theta)=0$ for $|\theta|>\theta_{F}$. For $|\theta|<\theta_{F}$, we have

$$
\rho(\theta)=\frac{\Delta}{2 \pi} \cosh (\theta)+\int_{-\theta_{F}}^{\theta_{F}} d \theta^{\prime} \rho\left(\theta^{\prime}\right) \Gamma_{++}\left(\theta-\theta^{\prime}\right),
$$

where

$$
\Gamma_{++}(\theta) \equiv \frac{1}{2 \pi i} \partial_{\theta} \log S_{++}(\theta)=\frac{1}{\theta^{2}+\pi^{2}} .
$$

The first term on the right-hand-side of (22) is the free term, while the kernel, $\Gamma_{++}$, appearing in the second term measures the strength of the interactions. As $\Gamma_{++}>0$, the interactions lead to a density of states greater than the bare value of $\frac{\Delta}{2 \pi} \cosh (\theta)$ associated with purely free fermions. The strength of the magnetic field, $H$, determines the Fermi rapidity, $\theta_{F}$. To find it, it is first convenient to introduce the dressed energy, $\epsilon(\theta)$, of the magnons. This is the amount of energy the system loses when a particle of rapidity, $\theta$, is removed from the sea. It is given by

$$
\epsilon(\theta)=\Delta \cosh (\theta)-H+\int_{-\theta_{F}}^{\theta_{F}} d \theta^{\prime} \epsilon\left(\theta^{\prime}\right) \Gamma_{++}\left(\theta-\theta^{\prime}\right) .
$$

At zero temperature, the particles fill all allowed levels up to $\theta=\theta_{F}$. Therefore the Fermi rapidity is determined by solving (24) subject to the boundary condition,

$$
\epsilon\left(\theta_{F}\right)=0
$$

Thus for $|\theta|<\theta_{F}, \epsilon(\theta)<0$. The energy of the system per unit length at zero temperature is given by

$$
E(H)=\int_{-\theta_{F}}^{\theta_{F}} \rho(\theta)[\Delta \cosh (\theta)-H]=\Delta \int_{-\theta_{F}}^{\theta_{F}} \frac{d \theta}{2 \pi} \epsilon(\theta) \cosh (\theta) .
$$

The zero-temperature magnetization follows immediately from the above equation,

$$
M(H)=-\partial_{H} E(H),
$$

with the corresponding susceptibility given by $\chi(H)=\partial_{H} M(H)$.

We now are able to compute the Luttinger parameters $K$ and $v_{F}$ appearing in the effective theory as functions of $H / \Delta$. The key is to study this system in terms of its gapless quasi-particle excitations present when $H>\Delta$. These excitations are not the original magnons, but rather the excitations above the magnon sea. Since they are gapless, they move either to the right or left at the Fermi velocity. We define their rapidity $\theta$ via $\epsilon(\theta)= \pm v_{F} p(\theta)=\mu e^{ \pm \theta}$, where the mass scale $\mu$ is arbitrary and can be redefined by a shift in $\theta$. To compute $v_{F}$ for the gapless excitations above the magnon sea, we first note that

$$
v_{F}=\left.\frac{\partial \epsilon}{\partial p}\right|_{\epsilon=\epsilon_{F}}=\left.\frac{\partial \theta}{\partial p} \frac{\partial \epsilon}{\partial \theta}\right|_{\theta=\theta_{F}} .
$$




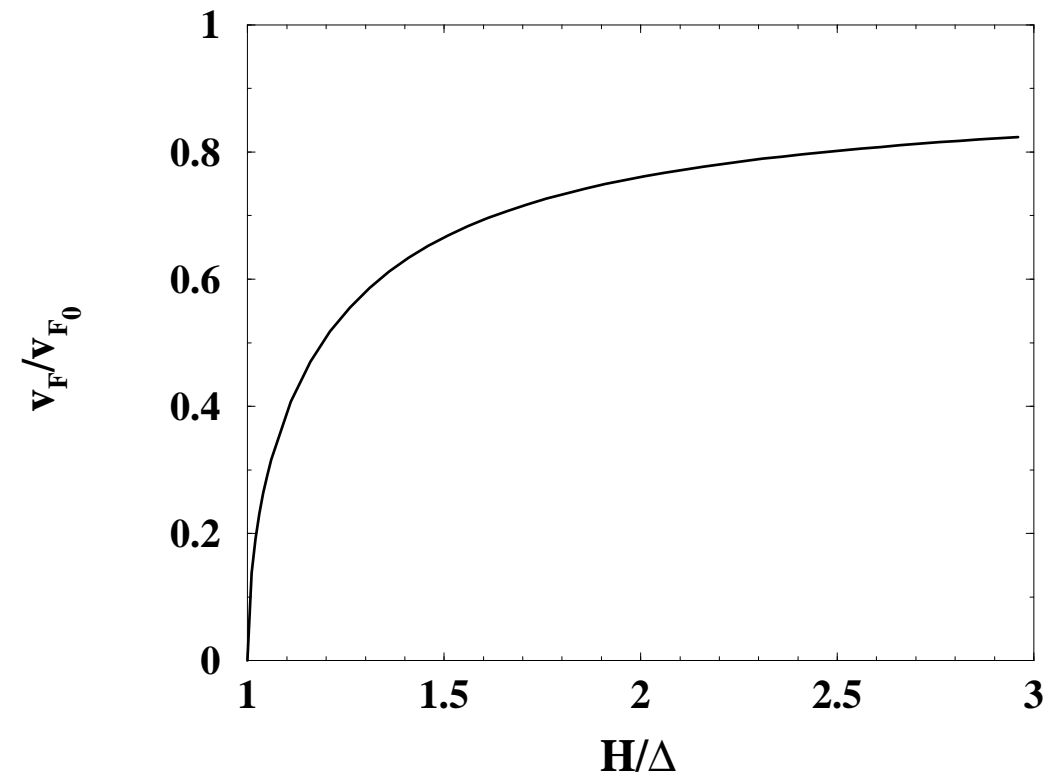

Figure 2: A plot of the Fermi velocity of the low-energy excitations present in the $S_{z}=1$ band when $H>\Delta$.

Here $p$ is the dressed momentum of the excitations and is given by an equation similar to the one governing the energy:

$$
p(\theta)=\Delta \sinh (\theta)+\int_{-\theta_{F}}^{\theta_{F}} d \theta^{\prime} \rho\left(\theta^{\prime}\right) \phi\left(\theta-\theta^{\prime}\right),
$$

where $\phi(\theta)=\log S_{++}(\theta) / 2 \pi i$ is the unrenormalized scattering phase. By comparing (28) with (22) we see $\partial_{\theta} p=2 \pi \rho$. The Fermi velocity is then given by

$$
v_{F}=\left.\frac{1}{2 \pi \rho(\theta)} \frac{\partial \epsilon}{\partial \theta}\right|_{\theta=\theta_{F}} .
$$

While the integral equations (22) and 24) cannot be solved in closed form, it is easy to solve numerically. This yields the curve plotted in Figure 2. Its most notable feature is the vanishing of $v_{F}$ for $H$ near $\Delta$. Below we will give a power-series expansion for $v_{F}$, valid when $H$ is near $\Delta$.

The coupling $K$ is similarly straightforward to find. As we now show, it is related to the renormalized spin, $S_{R}$, of excitations near the Fermi surface. $S_{R}$ measures how interactions change the response of a spin $S_{z}=1$ excitation near the Fermi surface to a change in the magnetic field. It is related to the dressed energy by

$$
S_{R}=-\left.\partial_{H} \epsilon(\theta)\right|_{\theta=\theta_{F}},
$$

and so obeys the integral equation,

$$
S_{R}=1+\int_{-\theta_{F}}^{\theta_{F}} S_{R}\left(\theta^{\prime}\right) \Gamma_{++}\left(\theta_{F}-\theta^{\prime}\right) .
$$




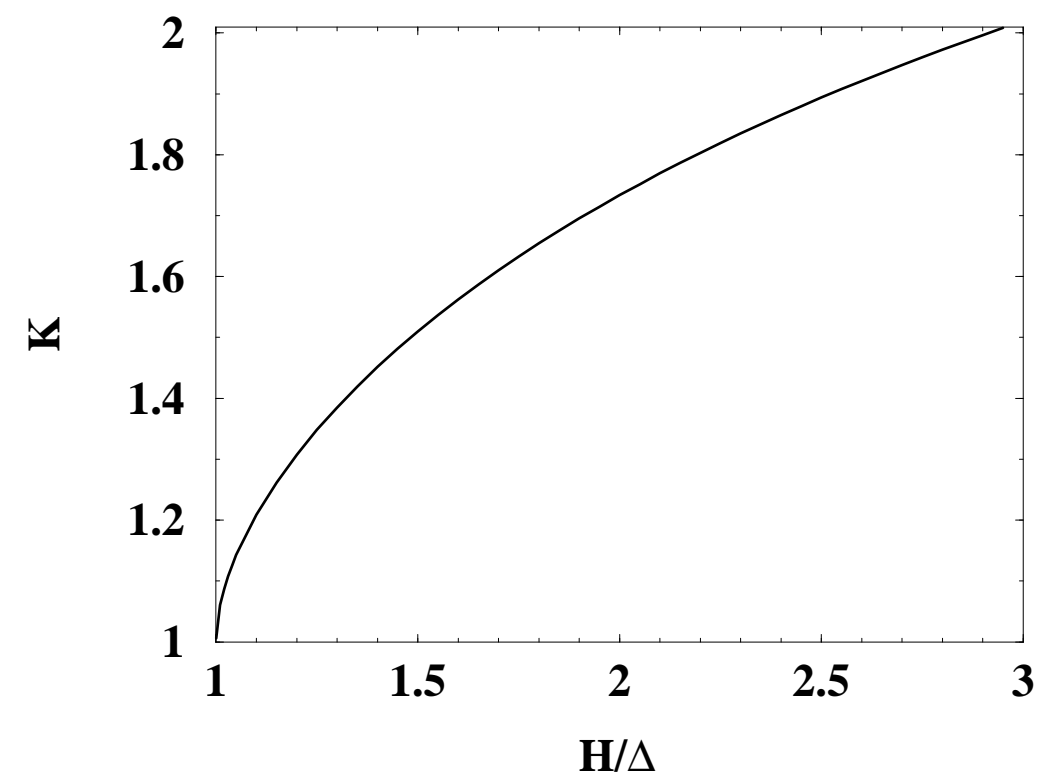

Figure 3: A plot of the Luttinger parameter describing the low energy excitations present in the $S_{z}=1$ band when $H>\Delta$.

The $H$ dependence of $S_{R}$ arises from the dependence of $\theta_{F}$ on $H$.

The basic idea behind the relationship of $K$ and $S_{R}$ is that the low-energy excitations near $\theta=\theta_{F}$ are free-fermionic. That is, their dressed S-matrices are -1 [25. In contrast to [25], here it is relatively easy to demonstrate this and we do so in Appendix B. As a consequence of the free-fermionic behavior, the only non-vanishing matrix elements of the current operator, $j_{L}=-2 K\left(v_{F}^{-1} \partial_{\tau}-i \partial_{x}\right) \phi_{L}$, involve a single particle-hole pair. Lorentz invariance requires that these matrix elements be given by

$$
\left\langle\theta_{h} \theta_{p}\left|j_{L}(0)\right| 0\right\rangle=\mu c e^{\left(\theta_{h}+\theta_{p}\right) / 2},
$$

where $\theta_{h}$ and $\theta_{p}$ are the rapidities of the particle and hole respectively. The constant $c$ is simply related to both $K$ and $S_{R}$. In a Luttinger liquid, $K$ appears in the current-current correlator (18). To relate this to $c$, we insert a complete set of states and use the matrix element (32), giving

$$
\begin{aligned}
\left\langle j_{L}(x, t) j_{L}(0)\right\rangle & =\int \frac{d \theta_{p}}{2 \pi} \frac{d \theta_{h}}{2 \pi}\left\langle j_{L}(x, t) \mid \theta_{h} \theta_{p}\right\rangle\left\langle\theta_{p} \theta_{h} \mid j_{L}(0)\right\rangle \\
-\frac{K}{\left(i x+v_{F} \tau\right)^{2}} & =\frac{c^{2} \mu^{2}}{4 \pi^{2}} \int d \theta_{h} d \theta_{p} e^{-\mu\left(i x+v_{F} \tau\right)\left(e^{\left.\theta_{h}+e^{\theta_{p}}\right)} e^{\theta_{h}+\theta_{p}}\right.} \\
& =\frac{c^{2}}{4 \pi^{2}\left(i x+v_{F} \tau\right)^{2}} .
\end{aligned}
$$

On the other hand, $c$ is related to $S_{R}$, as integrating the above matrix element gives the value of $S_{R}$ on the one-particle states:

$$
2 \pi S_{R} \delta\left(\theta_{1}-\theta_{2}\right)=\int d x\left\langle\theta_{1}\left|M_{0}^{3}(x)\right| \theta_{2}\right\rangle=\int d x\left\langle\theta_{1}\left|\frac{j_{L}(x)}{2 \pi}\right| \theta_{2}\right\rangle
$$




$$
\begin{aligned}
& =-i \int \frac{d x}{2 \pi} \mu c e^{\left(\theta_{2}+\theta_{1}\right) / 2} e^{i \mu x\left(e^{\theta_{2}}-e^{\theta_{1}}\right)} \\
& =-i c \delta\left(\theta_{1}-\theta_{2}\right) .
\end{aligned}
$$

Thus $c=i 2 \pi S_{R}$ and so

$$
K=S_{R}^{2}
$$

Given $S_{R}$ is described by the integral equation (31), $K$ follows immediately. The results are plotted in Figure 3.

It is straightforward to find explicit power-series expansions for $K$ and $v_{F}$ valid for $H$ near $\Delta$. We begin by computing $\epsilon(\theta)$. For $H$ slightly larger than $\Delta$, we can expand $\epsilon(\theta)$ around $\theta=0$ :

$$
\epsilon(\theta)=d_{0}+d_{2} \theta^{2}+\mathcal{O}\left(\theta^{4}\right)
$$

Plugging this into (24) demands $d_{0}$ and $d_{2}$ satisfy

$$
\begin{aligned}
& d_{0}=(\Delta-H)\left(1+2 \Gamma_{++}(0) \theta_{F}\right)+\Delta \frac{\Gamma_{++}(0)}{3} \theta_{F}^{3}+\mathcal{O}\left(\theta_{F}^{4}\right) ; \\
& d_{2}=\frac{\Delta}{2}-2 \theta_{F} \Gamma_{++}^{2}(0)(\Delta-H)+\mathcal{O}\left(\theta_{F}^{4}\right),
\end{aligned}
$$

where $\Gamma_{++}(0)=1 / \pi^{2}$. To determine $\theta_{F}$ we apply the condition $\epsilon\left(\theta_{F}\right)=0$, resulting in

$$
\theta_{F}=\sqrt{2\left(\frac{H}{\Delta}-1\right)}+\frac{4}{3} \Gamma_{++}(0)\left(\frac{H}{\Delta}-1\right)+\mathcal{O}\left(\left(\frac{H}{\Delta}-1\right)^{3 / 2}\right) .
$$

Similarly we can show $\rho(\theta)$ to be

$$
\rho(\theta)=\rho_{0}+\rho_{2} \theta^{2}
$$

where

$$
\begin{aligned}
& 2 \pi \rho_{0}=\Delta\left(1+2 \Gamma_{++}(0) \theta_{F}+4 \theta_{F}^{2} \Gamma_{++}^{2}(0)\right)+\mathcal{O}\left(\theta_{F}^{3}\right) ; \\
& 2 \pi \rho_{2}=\frac{\Delta}{2}-2 \theta_{F} \Gamma_{++}^{2}(0) \Delta+\mathcal{O}\left(\theta_{F}^{2}\right) .
\end{aligned}
$$

We can find the renormalized spin similarly.

These expansions give the power-series expansions for $K$ and $v_{F}$ to be

$$
\begin{aligned}
& K=1+\frac{2^{5 / 2}}{\pi^{2}}\left(\frac{H}{\Delta}-1\right)^{1 / 2}+\frac{88}{3 \pi^{4}}\left(\frac{H}{\Delta}-1\right)+\mathcal{O}\left(\frac{H}{\Delta}-1\right)^{3 / 2} \\
& v_{F}=v_{F_{0}}\left[\sqrt{2}\left(\frac{H}{\Delta}-1\right)^{1 / 2}-\frac{8}{3 \pi^{2}}\left(\frac{H}{\Delta}-1\right)+\mathcal{O}\left(\frac{H}{\Delta}-1\right)^{3 / 2}\right] .
\end{aligned}
$$

We have restored the bare spin-wave velocity $v_{F_{0}}$ (earlier set to one). 
We see that as a function of the magnetic field, the Luttinger parameter $K$ is 1 at threshold $H=\Delta$, and then increases with increasing field strength. That $K=1$ at $H=\Delta$ is a universal result already obtained by [26, 27]. It is independent of the exact nature of the excitations that fill the ground state at finite magnetization. In the low magnetization regime, the excitations of the ground state interact only weakly due to their low density. Thus to a zeroth order approximation, they can be treated as free fermions with a corresponding Luttinger parameter of $K=1$. Thus in the analysis of antiferromagnetic spin ladders [31], $K=1$ at $H=\Delta$ is again found even though $K$ decreases (opposite to the behaviour of the spin-1 chains) with subsequent increases in $H$. Similarly in mapping the spin chain onto an interacting Bose gas [34, one arrives at $K=1$ for $H \sim \Delta$. Here however $K$ at least increases with increasing field. By fine tuning the strength of the interactions in the gas, one could in principle come close to reproducing the behaviour as predicted by the O(3) NLSM.

We illustrate this in more detail. The equations governing the energy and renormalized spin of the Bose gas are 35] (in the case of the Bose gas the quantity equivalent to $S_{R}$ is the renormalized charge)

$$
\begin{aligned}
\epsilon(\theta) & =\theta^{2}-H+\int_{-\theta_{F}}^{\theta_{F}} d \theta^{\prime} \epsilon\left(\theta^{\prime}\right) \Gamma_{++}\left(\theta-\theta^{\prime}\right) ; \\
S_{R} & =1+\int_{-\theta_{F}}^{\theta_{F}} S_{R}\left(\theta^{\prime}\right) \Gamma_{++}\left(\theta_{F}-\theta^{\prime}\right) ; \\
\Gamma_{++}(\theta) & =\frac{c}{\pi} \frac{1}{c^{2}+\theta^{2}} .
\end{aligned}
$$

Here $c$ is the strength of the interactions in the Bose gas. If $c=\pi$, we see the equations of the Bose gas are nearly identical to those of the $O(3)$ NLSM. The sole difference (apart from some trivial shifts and rescalings) lies in the dependence of the bare energy upon $\theta$, i.e. $\epsilon_{0}^{\text {Bose }}(\theta)=\theta^{2}-H$ while $\epsilon_{0}^{\mathrm{NLSM}}(\theta)=\Delta \cosh (\theta)-H$. At low energies the two are identical, but diverge at higher scales.

\section{Scaling Behavior at $H>\Delta$}

Using the results for $K$ and $v_{F}$ derived in the last section, we describe the scaling behavior present in the magnetized phase of the spin chain.

\subsection{Susceptibility and Spin Conductance}

We first consider the computation of the magnetic susceptibility. To take into account the effects of a magnetic field we add a term to the Lagrangian of the form

$$
\begin{aligned}
\delta \mathcal{L} & =H \int d x d t M_{z} \\
& =H \int d x d t \frac{K}{\pi v_{F}} \partial_{t} \Phi .
\end{aligned}
$$


Here we have used that the oscillating term of $M_{z}$ vanishes with the integration over $x$. Doing so the relevant correlator in computing the susceptibility is then

$$
\left\langle M_{z} \partial_{t} \Phi\right\rangle \text {. }
$$

This correlator can be evaluated in the bosonic formulation of the Luttinger liquid giving

$$
\left\langle M_{z}(x, \tau) \frac{K}{\pi v_{F}} \partial_{t} \Phi(0)\right\rangle=\frac{K T^{2}}{4 v_{F}^{2}}\left[\frac{1}{\sinh ^{2}\left(\frac{i \pi T}{v_{F}} z\right)}+\frac{1}{\sinh ^{2}\left(\frac{i \pi T}{v_{F}} \bar{z}\right)}\right] .
$$

Here we have evaluated it at finite temperature. By taking the appropriate analytical continuations, we can arrive at the retarded correlator necessary to compute the susceptibility, $\chi(H)$. We so find

$$
\chi\left(H>H_{c}\right)=\left.\left\langle M_{z} \frac{K}{\pi v_{F}} \partial_{t} \Phi\right\rangle_{\text {retarded }}\right|_{\omega=0, k=0}=\frac{K(H)}{\pi v_{F}(H) .}
$$

We point out that the static susceptibility is independent of temperature.

As we are able to compute the susceptibility of the spin chain directly from the thermodynamics of the $O(3)$ NLSM, we are able to perform a non-trivial check on the correctness of this calculation. The susceptibility, $\chi$, at $\mathrm{T}=0$ is given by taking two derivatives of the energy, as displayed in (25) and (26). Expanding this in a power series as explained at the end of the last section gives the energy per unit length to be

$$
E(H)=-\frac{\Delta^{2}}{2 \pi}\left[\frac{2^{5 / 2}}{3}\left(\frac{H}{\Delta}-1\right)^{3 / 2}+\frac{16}{3 \pi^{2}}\left(\frac{H}{\Delta}-1\right)^{2}+\mathcal{O}\left(\frac{H}{\Delta}-1\right)^{5 / 2}\right]
$$

so that the susceptibility is

$$
\chi(H)=-\partial_{H}^{2} E(H)=\frac{\sqrt{2}}{2 \pi}\left(\frac{H}{\Delta}-1\right)^{-1 / 2}+\frac{16}{3 \pi^{3}}+\mathcal{O}\left(\frac{H}{\Delta}-1\right)^{1 / 2}=\frac{K(H)}{\pi v_{F}(H)} .
$$

We see that we recover the susceptibility as derived from the above Kubo formula.

We can also compute the spin conductivity. The spin conductivity measures the resulting spin current arising from a spatially varying magnetic field and is defined via

$$
j_{s}=\sigma_{s} \nabla H \text {. }
$$

The spin current operator has the general form

$$
j_{s}=-v_{F} \frac{K}{\pi} \partial_{x} \Phi+\text { term dependent upon } M .
$$

However the latter term in $j_{s}$ involving $M$ will not couple to $\delta \mathcal{L}$ in (42). As such the spin conductance is given simply from a Kubo formula,

$$
\begin{aligned}
\operatorname{Re} \sigma_{s}(\omega, H) & =\lim _{k \rightarrow 0} \frac{1}{k} \operatorname{Im}\left\langle j_{s}\left(-\frac{K}{v_{F} \pi} \partial_{t} \Phi\right)\right\rangle_{\text {ret }}(\omega, k) \\
& =\lim _{k \rightarrow 0} \frac{K^{2}}{\pi^{2}} \frac{1}{k} \operatorname{Im}\left\langle\partial_{x} \Phi \partial_{t} \Phi\right\rangle_{\mathrm{ret}}(\omega, k) \\
& =v_{F} K(H) \delta(\omega) .
\end{aligned}
$$

This result is valid at any temperature within the Luttinger liquid framework. 

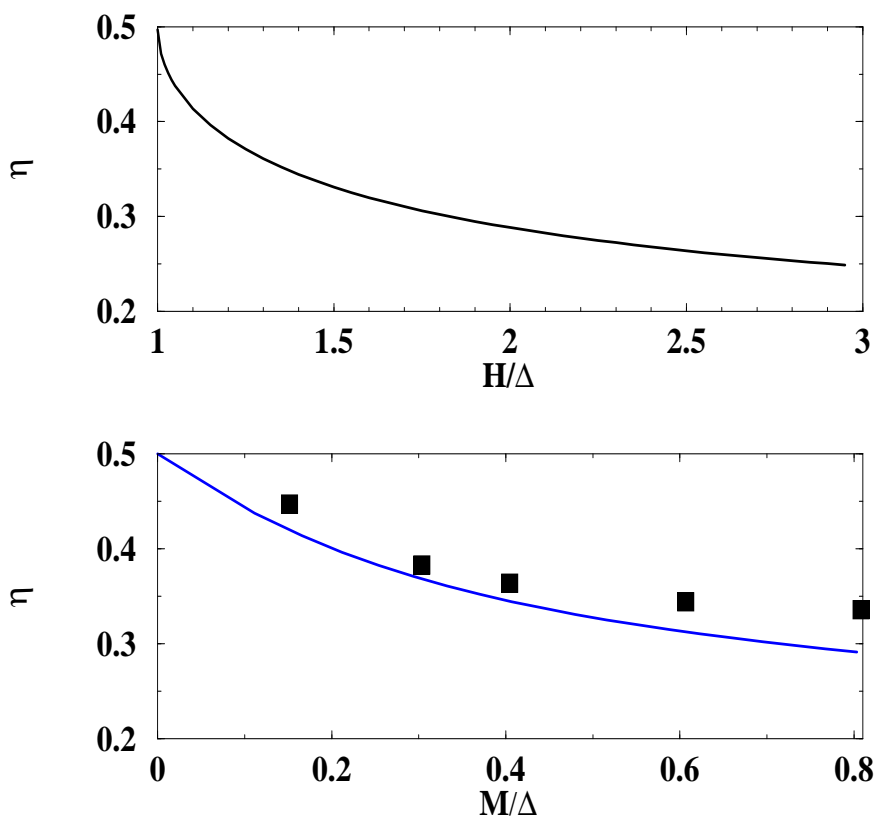

Figure 4: Plots of the scaling exponent $\eta$ for the transverse spin-spin correlator, $\left\langle S_{x}(x) S_{x}(0)\right\rangle \sim$ $|x|^{-\eta}$. In the top panel $\eta$ is plotted versus the applied magnetic field while in the bottom panel the $\eta$ is plotted versus the magnetization of the ground state. In this latter graph we also plot the values (squares) of $\eta$ found in Campos Venuti et al. through a density matrix renormalization group analysis.

\subsection{Inelastic Neutron Scattering}

We can make a series of predictions relevant for inelastic neutron scattering experiments with $H>H_{c}$. For scattering near wave-vector $k=\pi$, the experiments probe the single particle spectral weight of the field, $n^{a}(x, t)$. The scattering cross section is given in terms of the correlation function,

$$
\sigma(\omega, k) \propto \int d \omega d k e^{-i \omega t+i k x} \sum_{a}\left\langle n^{a}(x, t) n^{a}(0,0)\right\rangle .
$$

Because $n_{3}$ is massive, its spectral weight is exponentially suppressed at low energies and temperatures and for such cases we need only consider the contribution of the correlator $\left\langle n^{+} n^{-}\right\rangle$:

$$
\sigma(\omega, k) \propto \int d x d t e^{-i \omega t+i k x}\left\langle n^{+}(x, t) n^{-}(0,0)\right\rangle .
$$

We first examine the $T=0$ behavior of this correlator.

From the relation (19), the correlator $\left\langle n^{+} n^{-}\right\rangle$equals

$$
\left\langle n^{+}(x, \tau) n^{-}(0,0)\right\rangle=\frac{1}{\left(x^{2}+v_{F}^{2} \tau^{2}\right)^{1 / 4 K}}+2 c_{1}^{2} \cos (2 \pi M x) \frac{1}{\left(x^{2}+v_{F}^{2} \tau^{2}\right)^{K+1 / 4 K}}
$$

The exponent, $\eta=1 / 2 K$, governing the leading piece of the transverse spin-spin correlator is plotted in the top panel of Figure 4 as a function of magnetic field. We see that its $H=\Delta^{+}$ 
value is $\eta=1 / 2$, equal to that of free fermions. For values of $H$ slightly larger than $\Delta, \eta=1 / 2 K$ takes the form

$$
\eta=\frac{1}{2 K}=\frac{1}{2}\left(1-\frac{2^{5 / 2}}{\pi^{2}}\left(\frac{H}{\Delta}-1\right)^{1 / 2}+\frac{8}{3 \pi^{4}}\left(\frac{H}{\Delta}-1\right)+\mathcal{O}\left(\frac{H}{\Delta}-1\right)^{3 / 2}\right) .
$$

In the bottom panel of Figure 4 we plot $\eta$ versus the magnetization of the ground state. We also compare our computation to that of numerical simulations of a lattice integer-spin chain, $H=J \sum_{i} S_{i} \cdot S_{i+1}$ done by Campos Venuti et al. [6]. The agreement between the two is reasonable but not overwhelming. At larger values of the magnetization, $M$, this is to be expected. For a lattice spin chain the magnetization must saturate at some critical value of the applied magnetic field, $H_{c} \sim J$. In contrast the magnetization of the $O(3)$ sigma model has no such upper bound. Thus we must work at values of the applied field where the magnetization of the system is small or alternatively, $H \ll J$. Given that $\Delta \sim .4 J$ in a spin 1 chain, this limit can only be ambiguously met at best. At smaller values of the magnetization, the disagreement may arise from differences between the lattice theory and its continuum version. Nonetheless, we find reasonable agreement.

At finite temperature we can easily determine the form of $\left\langle n^{+} n^{-}\right\rangle$. Via a conformal transformation we have,

$$
\begin{aligned}
\left\langle n^{+}(x, \tau) n^{-}(0,0)\right\rangle & =\left(\frac{\pi^{2}}{v_{F}^{2} \beta^{2}}\right)^{1 / 4 K} \frac{1}{\left|\sinh T \pi\left(x / v_{F}+i \tau\right)\right|^{1 / 2 K}} \\
+ & 2 c_{1}^{2} \cos (2 \pi M x)\left(\frac{\pi^{2}}{v_{F}^{2} \beta^{2}}\right)^{K+1 / 4 K} \frac{1}{\left|\sinh T \pi\left(x / v_{F}+i \tau\right)\right|^{2 K+1 / 2 K}} .
\end{aligned}
$$

Analytically continuing and then Fourier transforming thus gives us an expression for the cross section at finite $\mathrm{T}$,

$$
\begin{aligned}
& \sigma(\omega, k)=\frac{1}{2 v_{F}}\left(\frac{\pi}{\beta v_{F}}\right)^{-2+1 / 2 K} f_{1 / 4 K}\left(\frac{\beta}{\pi} \omega, \frac{\beta v_{F}}{\pi} k\right)+c_{1}^{2} \frac{1}{2 v_{F}}\left(\frac{\pi}{\beta v_{F}}\right)^{-2+K+1 / 4 K} \\
& \times\left(f_{K+1 / 4 K}\left(\frac{\beta}{\pi} \omega, \frac{\beta v_{F}}{\pi}(k+2 \pi M)\right)+f_{K+1 / 4 K}\left(\frac{\beta}{\pi} \omega, \frac{\beta v_{F}}{\pi}(k-2 \pi M)\right)\right),
\end{aligned}
$$

with $f_{\gamma}(x, y)$ equal to 41 ,

$$
\begin{aligned}
f_{\gamma}(x, y) & =h_{\gamma}\left(\frac{1}{2}(y-x)\right) h_{\gamma}\left(\frac{1}{2}(y+x)\right) \\
h_{\gamma}(x) & =\operatorname{Re}\left[(2 i)^{\gamma} B\left(\frac{\gamma-i x}{2}, 1-\gamma\right)\right],
\end{aligned}
$$

where $B$ is the beta function, $B(x, y)=\Gamma(x) \Gamma(y) / \Gamma(x+y)$. We so obtain a scaling form for $\sigma(\omega, k)$ with $f(x, y)$ a universal function. The scaling form two parts: one relevant for wavevectors $k$ near 0 and one yielding a contribution for $k$ near the incommensurate wave vector $2 \pi M$ (understanding that all wavevectors involving $n^{ \pm}$have been shifted by $\pi$ ).

We can analyze the above expression for $\sigma(\omega, k)$ in the small and large $T$ limits. In the case $T \ll M$ and $\omega, k \ll M$, we can safely ignore the contribution of the second term in (53). We then find

$$
\sigma(\omega, k) \sim \frac{2 \pi^{2}}{v_{F} \Gamma^{2}(1 / 4 K)} \Theta\left(-\omega-v_{F} k\right) \Theta\left(v_{F} k-\omega\right)\left(\frac{\omega^{2} / v_{F}^{2}-k^{2}}{16}\right)^{-1+1 / 4 K} ; \quad T \ll \omega, k ;
$$




$$
\begin{aligned}
\sigma(\omega, k) & =\frac{2}{v_{F}}\left(\frac{2 \pi}{\beta v_{F}}\right)^{-2+1 / 2 K} \cos ^{2}\left(\frac{\pi}{8 K}\right) B^{2}\left(\frac{1}{8 K}, 1-\frac{1}{4 K}\right) ; \quad T \gg \omega, k ; \\
& \sim T^{-2+1 / 2 K} .
\end{aligned}
$$

In the first case, $T \ll \omega, k$, the leading term is temperature independent. For the behavior in the second case to be observed, we need $\Delta \gg T$. Otherwise thermal excitations involving the other two bands, $\left(S_{z}=0,-1\right)$, would alter the Luttinger liquid behavior of the ground state. For wavevectors near $k \pm 2 \pi M$ the second term in (53) dominates and we find instead

$$
\begin{aligned}
\sigma(\omega, k \pm 2 \pi M) \sim c_{1}^{2} \frac{2 \pi^{2}}{v_{F} \Gamma^{2}(1 / 4 K+K)} \Theta\left(-\omega-v_{F} k\right) \Theta\left(v_{F} k+\omega\right) & \\
& \times\left(\frac{\omega^{2} / v_{F}^{2}-k^{2}}{16}\right)^{-1+K+1 / 4 K} ; T \ll \omega, k ; \\
\sigma(\omega, k \pm 2 \pi M)=c_{1}^{2} \frac{2}{v_{F}}\left(\frac{2 \pi}{\beta v_{F}}\right)^{-2+2 K+1 / 2 K} & \cos ^{2}\left(\pi\left(\frac{1}{8 K}+\frac{K}{2}\right)\right) \\
& \times B^{2}\left(\frac{1}{8 K}+\frac{K}{2}, 1-K-\frac{1}{4 K}\right) ; \quad T \gg \omega, k ; \\
\sim T^{-2+2 K+1 / 2 K} . &
\end{aligned}
$$

We thus see the power law dependencies of $\sigma(\omega, k)$ near $k \sim 2 \pi M$ differ from those near $k \sim 0$.

For scattering near the wave-vector, $k=0$, the spectral weight of the magnetization operator is probed. It is this operator that creates/destroys excitations near $k=0$. At low energies and temperatures the cross section, $\sigma$, is given by

$$
\sigma(\omega, k) \propto \int d t d x e^{-i \omega t+i k x}\left\langle M_{z}(x, t) M_{z}(0,0)\right\rangle .
$$

The other two spin components of the magnetization are massive and do not contribute at low energies. By the fluctuation-dissipation theorem, we can recast $\sigma$ in terms of the imaginary piece of the corresponding retarded correlator:

$$
\sigma(\omega, k) \propto-\frac{2}{1-e^{-\beta \omega}} \operatorname{Im}\left\langle M_{z} M_{z}\right\rangle_{\text {ret. }}(\omega, k) .
$$

In the case that $\omega, k \ll M$, we obtain

$$
\sigma(\omega, k) \propto \frac{K}{v_{F}} \frac{\omega}{1-e^{-\beta \omega}}\left(\delta\left(\omega+v_{F} k\right)+\delta\left(\omega-v_{F} k\right)\right) .
$$

For wavevectors near $2 \pi M$ we find instead

$$
\begin{gathered}
\sigma(\omega, k \pm 2 \pi M) \sim-\frac{c^{2}}{1-e^{-\beta \omega}} \Gamma^{2}(1-K) \sin (\pi(K-1)) \frac{\sin (\pi K)}{v_{F}}\left(\frac{\omega^{2}-k^{2}}{4}\right)^{K-1} \\
\times(\operatorname{sgn}(w+k)+\operatorname{sgn}(w-k)) ; \quad T \ll \omega, k ; \\
\sigma(\omega, k \pm 2 \pi M) \sim-\frac{2 c^{2} \omega}{1-e^{-\beta \omega}}\left(\frac{2 \pi}{\beta v_{F}}\right)^{2 K-3} \frac{\sin (\pi K)}{v_{F}} B^{2}\left(\frac{K}{2}, 1-K\right) \psi(K / 2) ; T \gg \omega, k ;
\end{gathered}
$$

where $\psi(x)=\partial_{x} \log (\Gamma(x))$. 


\subsection{NMR Relaxation Rates}

As with the inelastic neutron scattering cross section, we can make a series of predictions for NMR relaxation rates. The NMR relaxation rate is given in terms of the spin-spin correlation function [18]:

$$
\frac{1}{T_{1}}=\sum_{\substack{\alpha=1,2 \\ \beta=1,2,3}} \int \frac{d k}{2 \pi} A_{\alpha \beta}(k) A_{\alpha \gamma}(-k)\left\langle n^{\beta} n^{\gamma}\right\rangle\left(k, \omega_{N}\right),
$$

where $\omega_{N}=\gamma_{N} H$ is the nuclear Lamour frequency with $\gamma_{N}$ the nuclear gyromagnetic ratio and the $A_{\alpha \beta}$ are the hyperfine coupling constants. In the above we have assumed $H$ is aligned in the 3(z)-direction. We assume that the hyperfine couplings are independent of the wavevector $k$. Hence

$$
\frac{1}{T_{1}} \propto A_{\alpha \beta} A_{\alpha \gamma}\left\langle n^{\beta} n^{\gamma}\right\rangle\left(x=0, \omega_{N} \sim 0\right) .
$$

We now consider the correlator $\langle n n\rangle$ in more detail. Specifically we will compute the contributions to $T_{1}^{-1}$ coming from the different branches of low energy excitations.

If the hyperfine couplings are such that the transverse fields are dominant we obtain for $1 / T_{1}$,

$$
\begin{aligned}
\frac{1}{T_{1}} & \sim \lim _{\omega \rightarrow 0} \int d t e^{i \omega t}\left\langle n_{+}(t, x=0) n_{-}(0)\right\rangle \\
& =\lim _{\omega \rightarrow 0} \frac{1}{2 v_{F}}\left(\frac{\pi}{\beta v_{F}}\right)^{-1+1 / 2 K} h_{1 / 2 K}(\beta \omega / \pi)+\frac{c_{1}^{2}}{v_{F}}\left(\frac{\pi}{\beta v_{F}}\right)^{-1+2 K+1 / 2 K} h_{1 / 2 K+2 K}(\beta \omega / \pi),
\end{aligned}
$$

where $h_{1 / 2 K}$ is as defined in (54). In the low temperature limit the second term is subdominant and we have

$$
\frac{1}{T_{1}} \propto \frac{1}{2 v_{F}}\left(\frac{\pi}{\beta v_{F}}\right)^{-1+1 / 2 K} h_{1 / 2 K}(0) .
$$

In the low density limit (i.e. $H \sim \Delta^{+}$), we have $1 / T_{1} \sim T^{-1 / 2}$. In this limit $1 / T_{1}$ has a dependence upon $T$ identical to that of antiferromagnetic ladders [31, 32]. However as $H$ is increased, the power law dependencies of the two cases diverge.

If on the other hand the hyperfine couplings are such that the contribution from $\left\langle M_{z} M_{z}\right\rangle$ is important we find,

$$
\begin{aligned}
\frac{1}{T_{1}} & \sim \lim _{\omega \rightarrow 0} \int d t e^{i \omega t}\left\langle M_{z}(t, x=0) M_{z}(0)\right\rangle, \\
& =\frac{2 K T}{v_{F}^{2}}+c^{2}\left(\frac{\pi}{\beta v_{F}}\right)^{2 K-1} h_{2 K}(0) .
\end{aligned}
$$

As $K>1$ for any finite $\mathrm{H}$ in excess of $\Delta$, the first of the two terms dominates.

\subsection{Validity of Luttinger Liquid Picture}

The Luttinger liquid picture we have developed is precise only at zero temperature. Nonetheless we have argued that this behavior will persist to some degree at finite temperature. We are in position to analyze qualitatively at least whether this is indeed true. To this end, we compute the susceptibility at finite temperature and finite magnetic field. We do so using a more sophisticated 


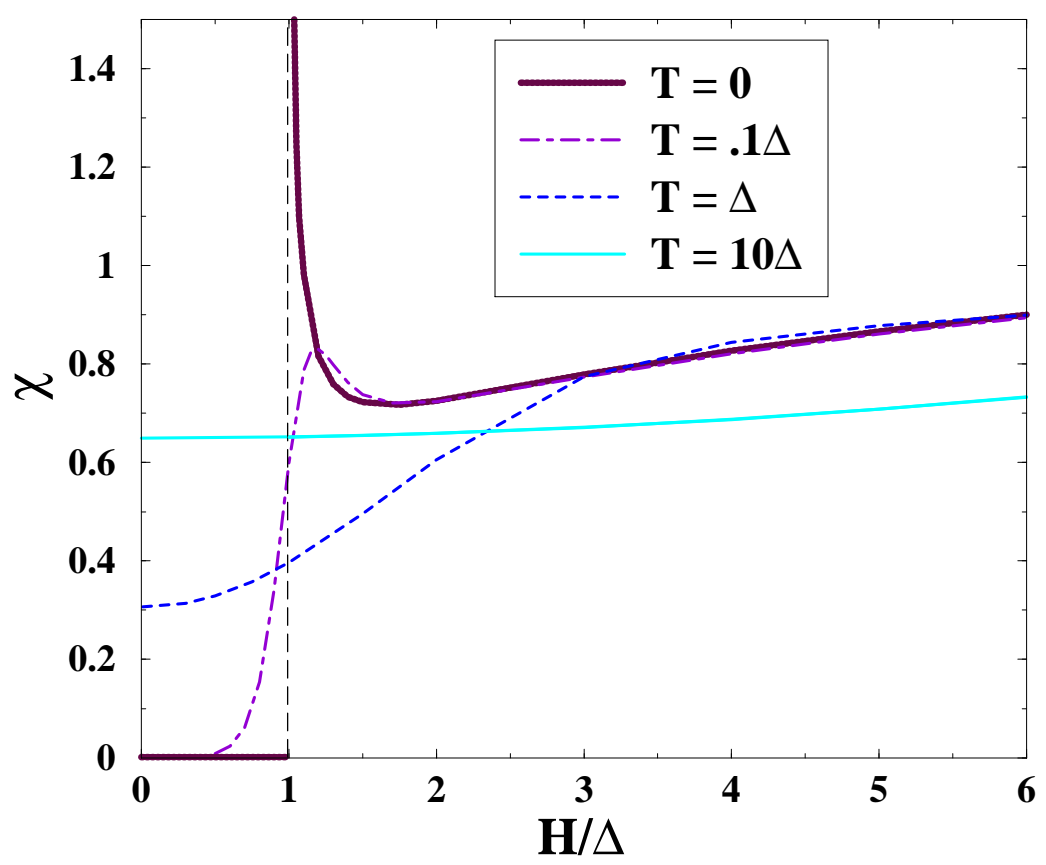

Figure 5: Plots of the susceptibility as a function of applied field for a variety of temperatures.

formalism than presented previously: the thermodynamic Bethe ansatz. For the $O(3)$ sigma model, the appropriate equations were originally given in [39] but can also be found in [19, 40]. ${ }^{1}$

Plots of the exact susceptibility for various temperatures derived from these equations are given in Figure 5. Note in particular how the peak at $H=\Delta$ appears as the temperature is lowered. At $T=0$, we see from (46) that this turns into a square-root singularity. The divergence of the susceptibility from its $T=0$ value is a reasonable indication of the persistence of the Luttinger-liquid picture at finite temperature as $\chi$ can be given directly in terms of $K$ and $v_{F}$ (see (24)). Thus we expect finite temperatures to be destructive of Luttinger liquids associated with fields, $H$, only slightly in excess of the gap where a finite temperature drastically rounds off the square root singularity appearing in $\chi$. However at higher fields where the density of magnons in the ground state is larger and so presumably more robust against small temperature perturbations, the susceptibility is equal to its zero temperature value. In this case we expect the $T=0$ Luttinger liquid picture to remain valid.

We note as an aside the square root singularity in $\chi$ appearing in Figure 5 at $T=0$ is a generic feature. It appears in any model (not only spin chains) with a gap and a low-energy

\footnotetext{
${ }^{1}$ Apropos of nothing, we have also computed the susceptibility at large temperatures but zero field. In the zero-field large- $T$ limit, Sachdev and Damle [21] provide a high temperature computation of the susceptibility predicated on integrating out higher Matsubara frequencies. To subleading order it is given by

$$
\chi(T)=\frac{1}{3 \pi}\left(\log \left(\frac{32 \pi e^{-2-\gamma} T}{\Delta}\right)+\log \left(\log \left(\frac{8 T}{e \Delta}\right)\right)+\mathcal{O}\left(\frac{\log (\log (T))}{\log (T)}\right) .\right.
$$

From a numerical analysis of the TBA equations at temperatures in the range, $T \sim 10^{3} \Delta-10^{7} \Delta$, we find agreement with this analytical form at the $1 \%$-level. To obtain this agreement it was important to include the subleading term.
} 


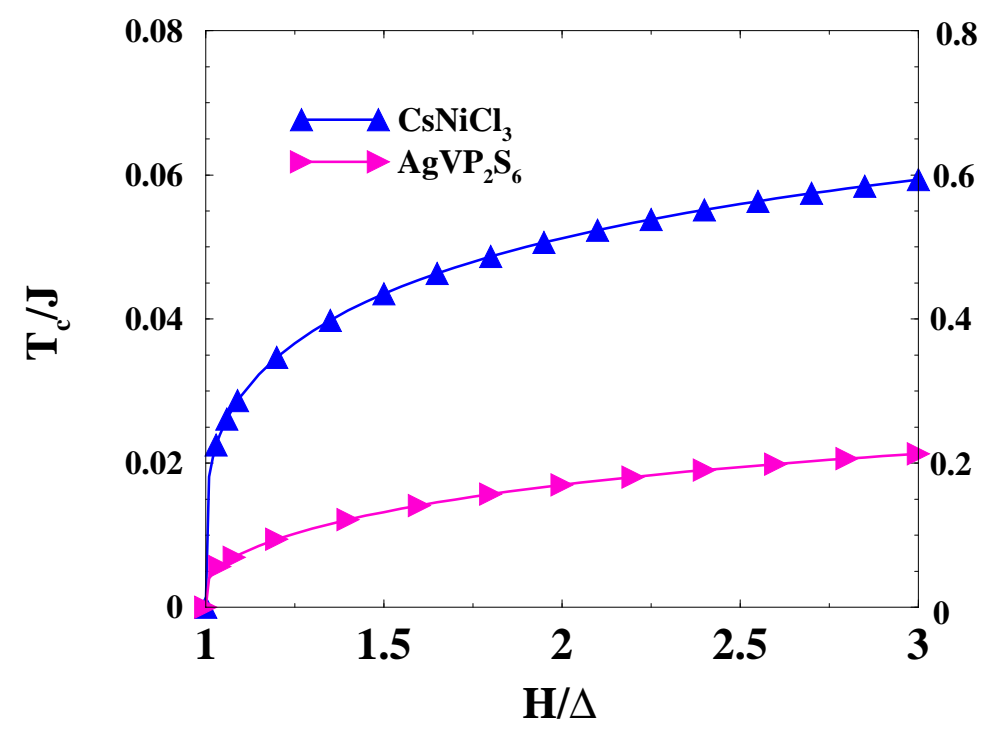

Figure 6: A plot of the dependence of the 3D ordering temperature, $T_{c} / J$, as a function of applied field, $H$, for two different spin chain compounds. The relevant values of $J$ for two materials are $J_{\mathrm{CsNiCl}_{3}} \sim 16 \mathrm{~K}$ and $J_{\mathrm{AgVP}_{2} \mathrm{~S}_{6}} \sim 320 \mathrm{~K}$. The corresponding values of inter to intrachain anisotropies are $J^{\prime} / J \sim .017\left(\mathrm{CsNiCl}_{3}\right)$ and $J^{\prime} / J \sim 10^{-5}\left(\mathrm{AgVP}_{2} \mathrm{~S}_{6}\right)$. The left ordinate scale corresponds to $\mathrm{AgVP}_{2} \mathrm{~S}_{6}$ while the right scale corresponds to $\mathrm{CsNiCl}_{3}$.

quadratic dispersion relation. Its appearance is not related in any way to the $O(3)$ NLSM being integrable (although the integrability allows us to compute exactly the coefficient of the divergence). We thus expect this square-root divergence to appear also in spin chains with large easy-axis anisotropies. Indeed the rounded finite temperature counterpart of this divergence has already been seen in NENP [37.

Beyond the effects of finite temperature, the Luttinger liquid phase can be destroyed by the presence of intrachain couplings. In mean field theory, three dimensional order due to such couplings develops at a transition temperature, $T_{c}$, given by

$$
1=\left|a J^{\prime} \chi_{\perp}\left(q=0, \omega=0, T=T_{c}\right)\right|,
$$

where $\chi_{\perp}$ is the staggered susceptibility of a single chain,

$$
\chi_{\perp}=\int d x d t e^{i \omega t-i k x}\left\langle n^{+}(x, t) n^{-}(0)\right\rangle_{\text {retarded }},
$$

and $J^{\prime}$ is the intrachain coupling. Here we explicitly include the lattice cutoff, $a^{-1} \sim J . q$ is the deviation from the wavevector, $\pi$. From the expressions for $n^{ \pm}$given in (19), we obtain, similar to [43,

$$
\begin{gathered}
\chi_{\perp}(q, w)=-\left(\frac{2 \pi a}{\beta v_{F}}\right)^{(-2+1 /(2 K))} \frac{\sin \left(\frac{\pi}{4 K}\right)}{v_{F}}\left[B\left(\frac{1}{8 K}-i \frac{v_{F} \omega_{-}}{4 \pi T}, 1-\frac{1}{4 K}\right) B\left(\frac{1}{8 K}-i \frac{v_{F} \omega_{+}}{4 \pi T}, 1-\frac{1}{4 K}\right)\right] \\
-c_{1}^{2}\left(\frac{2 \pi a}{\beta v_{F}}\right)^{\left.\left(-2+2 K+\frac{1}{2 K}\right)\right)} \frac{\sin \left(\pi\left(K+\frac{1}{4 K}\right)\right)}{v_{F}}\left[B\left(\frac{K}{2}+\frac{1}{8 K}-i \frac{v_{F}\left(\omega_{-}-2 \pi M\right)}{4 \pi T}, 1-K-\frac{1}{4 K}\right)\right.
\end{gathered}
$$




$$
\left.\times B\left(\frac{K}{2}+\frac{1}{8 K}-i \frac{v_{F}\left(\omega_{+}+2 \pi M\right)}{4 \pi T}, 1-K-\frac{1}{4 K}\right)+(M \rightarrow-M)\right],
$$

where $\omega_{ \pm}=\left(\omega / v_{F} \pm k\right)$. Combining this expression with the mean condition leads to a transition temperature given by 42 ]

$$
\begin{aligned}
\frac{T_{c}}{J} & =\frac{1}{\pi} \frac{v_{F}}{v_{F 0}}\left(\frac{1}{2} \frac{J^{\prime}}{J} \frac{v_{F 0}}{v_{F}} \delta\right)^{2 K /(4 K-1)} \\
\delta & \equiv \sin \left(\frac{\pi}{4 K}\right) B^{2}\left(\frac{1}{8 K}, 1-\frac{1}{4 K}\right)
\end{aligned}
$$

In deriving this expression we have ignored the term in $\chi_{\perp}$ appearing in (70) dependent upon $M$ whose contribution is negligible for small $T$.

Plotted in Figure 6 is the dependence of $T_{c}$ upon $H$ for the spin chain compounds $\mathrm{CsNiCl}_{3}$ and $\mathrm{AgVP} \mathrm{P}_{2} S_{6}$. We see that as $H$ is increased there is a corresponding increase in $T_{c}$. Thus at a fixed temperature and sufficiently large interchain coupling, $J^{\prime}$, we expect that as $H$ is increased there will be a transition to a state with long range order. We thus see that the Luttinger liquid phase is stable at a given temperature for smaller fields.

As discussed in the introduction, field induced long range order has been observed in the spin chain material, NDMAP [36]. Although this compound has a large single ion anisotropy, we make an attempt to compare experimental observations with our analysis. From inelastic neutron scattering experiments, the spin coupling, $J$, of NDMAP is $J=26.5 K$ [44. For a field applied along the chain (perpendicular to the easy plane defined by the anisotropy), the corresponding gap $\Delta$ is given in terms of $J$ and $D=.3 J$, the strength of the single ion anisotropy, by 45 ,

$$
\Delta \sim .4 J-.66 D=5.3 K=3.75 T \text {, }
$$

with the Landé $g$ factor equal to $g=2.1$. In order to obtain a reasonable fit with the experimental observations we take $J^{\prime} / J=2 \times 10^{-4}$. The results are plotted in Figure 7 . The value used for $J^{\prime} / J$ is considerably smaller (by a factor of 6) than the value employed in [36. These authors, however, were able to match the experimental data by assuming $\chi_{\perp}$ took the form $\chi_{\perp} \sim A T^{-2+1 / 2 K}$ with $A$ some field independent constant. We however find that $A$ is both field dependent and generally larger in magnitude than the value of $A$ used in [36. To compensate in matching the data, we need to take $J^{\prime} / J$ to be smaller in magnitude.

At fields not far in excess of the gap, we find reasonable agreement between the data and the theoretical computation. At larger values of the field, $H$, the computed $T_{c}$ exceeded the observed value. This is not surprising. As we saw in our computation of the exponent $\eta$, the assumptions underlying our computation fail to accurately describe the physics at large fields. With $H / \Delta>2$, we enter a region where the magnetization per site of the system is some significant fraction of its maximal value of 1 , a regime our method, which puts no bound upon the magnetization, is unable to handle properly.

The destruction of the Luttinger liquid phase due to an applied magnetic field occurs not only for spin-1 chains but for antiferromagnetic spin-1/2 ladders [31]. Unlike the result found in the case of two antiferromagnetically coupled spin-1/2 chains [31, we find however $T_{c}$ is strictly monotonic as a function of applied field. The difference in the two cases is again that we have $K>1$ and increasing with field while in the case of antiferromagnetic spin ladders, $K<1$ 


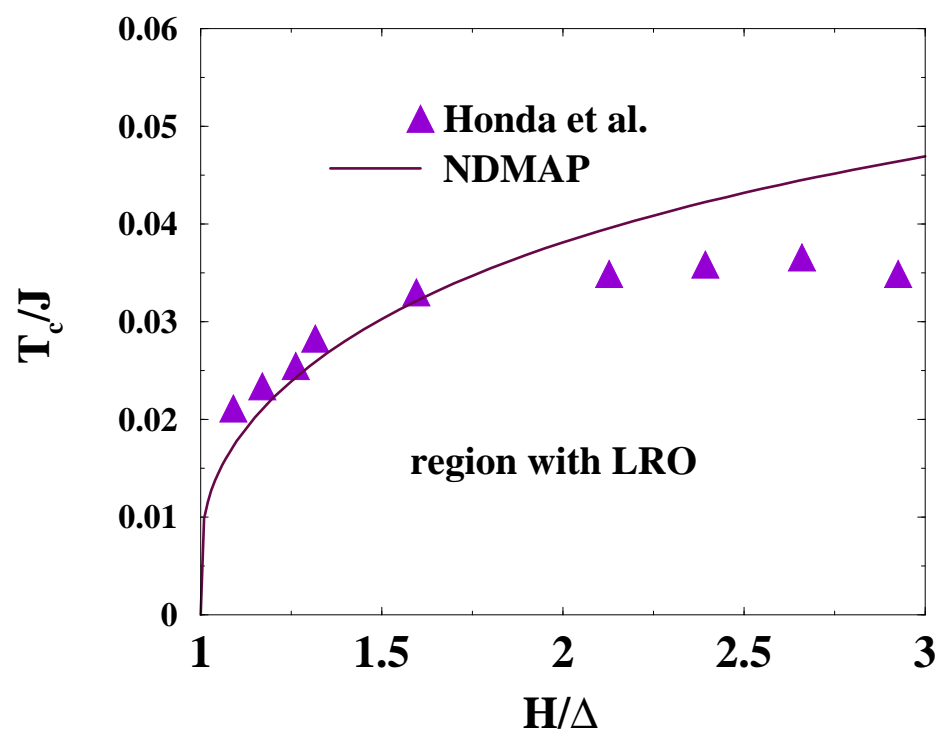

Figure 7: A plot of the dependence of the $3 \mathrm{D}$ ordering temperature, $T_{c} / J$, as a function of applied field, $H$, for the spin chain compound, NDMAP.

and decreasing with field. There is an additional instability that affects antiferromagnetic spin ladders, an instability to the formation of a spin-Peierls state due to coupling to the lattice [46]. However spin-1 chains are thought to be robust against this perturbation [46]. 


\section{A Analysis of the Ground State at $\mathbf{T}=0$}

In this section we determine the characteristics of the zero temperature ground state in a magnetic field, $H$, exceeding the gap. At the heart of this analysis lies the insistence that the ground state wave function be compatible with the scattering amplitude, $S_{++}$, as defined in (21). This amplitude characterizes the wave function, $\psi\left(\theta_{1}, \ldots, \theta_{N}\right)$, of a ground state with $N$ particles through the application of periodic boundary conditions. In allowing the i-th particle, $\theta_{i}$, to traverse the entire length, $L$, of the system and so commute with the other $N-1$ particles, the wave function picks up a phase

$$
\begin{aligned}
\psi\left(\theta_{1}, \ldots, \theta_{N}\right) & \rightarrow \phi\left(\theta_{i}\right) \times \psi\left(\theta_{1}, \ldots, \theta_{N}\right) \\
\phi\left(\theta_{i}\right) & =\prod_{j \neq i}^{N} S_{++}\left(\theta_{i}-\theta_{j}\right) e^{i L \Delta \sinh \left(\theta_{i}\right)} .
\end{aligned}
$$

The first term is a product of scattering amplitudes arising from the i-th particle scattering with the remaining $N-1$ particles. The second term comes from the bare momentum carried by the i-th particle. With periodic boundary conditions, we must have

$$
\phi\left(\theta_{i}\right)=1
$$

Taking logarithms of this constraint leads to the quantization condition,

$$
N_{i}=\frac{L \Delta}{2 \pi} \sinh \left(\theta_{i}\right)+\sum_{j \neq i} \frac{1}{2 \pi i} \log S_{++}\left(\theta_{i j}\right),
$$

where $N_{i}$ is the quantum number characterizing the rapidity, $\theta_{i}$, (i.e. $\log (1)=i 2 \pi N_{i}$ ). As we approach the continuum limit, we can then write a difference equation between successive integers, $N_{i}$ and $N_{i+1}=N_{i}+1$,

$$
1=\frac{L \Delta}{2 \pi} \cosh \left(\theta_{i}\right) \delta \theta_{i}+\sum_{j \neq i} \frac{1}{2 \pi i}\left(\partial_{\theta} \log S_{++}\right)\left(\theta_{i j}\right) \delta \theta_{i} ; \quad \delta \theta_{i}=\theta_{i+1}-\theta_{i} .
$$

The density of states per unit length at $\theta_{i}$ is defined as $n\left(\theta_{i}\right) \equiv 1 / L \delta \theta_{i}$. With this we can rewrite the above in the continuum limit as,

$$
\rho(\theta)+\tilde{\rho}(\theta)=\frac{\Delta}{2 \pi} \cosh (\theta)+\int d \theta^{\prime} \rho\left(\theta^{\prime}\right) \Gamma_{++}\left(\theta-\theta^{\prime}\right)
$$

where $\rho$ is the density of occupied states and $\tilde{\rho}$ is the density of unoccupied states, so that $\rho+\tilde{\rho}=n$.

At zero temperature, the equation for $\rho$ can be simplified. With $T=0$, the ground state has a Fermi surface at $\theta_{F}$. Excitations with rapidities, $|\theta|>\theta_{F}$, do not appear in the ground state. Thus

$$
\rho(\theta)= \begin{cases}n(\theta), & |\theta|<\theta_{F} \\ 0, & |\theta|>\theta_{F}\end{cases}
$$




$$
\tilde{\rho}(\theta)=\left\{\begin{array}{cc}
0, & |\theta|<\theta_{F} \\
n(\theta), & |\theta|>\theta_{F}
\end{array}\right.
$$

and we obtain equation (22) for $\rho$ at zero temperature.

We have computed the interacting density of states. We can also compute the interacting energy of the $S_{z}=1$ excitations in the ground state. The total energy of the system equals

$$
E=\int d \theta(\Delta \cosh (\theta)-H) \rho(\theta)
$$

If we vary the density of occupied particles and holes, $\rho \rightarrow \rho+\delta \rho$ and $\tilde{\rho} \rightarrow \tilde{\rho}+\delta \tilde{\rho}$, the total energy varies accordingly

$$
\delta E=\int d \theta(\Delta \cosh (\theta)-H) \delta \rho(\theta)
$$

But we can also express this variation in energy in terms of the interacting or dressed energies of the excitations:

$$
\delta E=\int d \theta\left(\delta \rho(\theta) \epsilon^{+}(\theta)-\delta \tilde{\rho}(\theta) \epsilon^{-}(\theta)\right)
$$

$\epsilon^{+}(\theta) / \epsilon^{-}(\theta)$ mark the energies needed to excite a particle/hole above the ground state. At zero temperature they are defined such that

$$
\begin{aligned}
& \epsilon^{+}(\theta)=\left\{\begin{array}{ll}
>0, & |\theta|>\theta_{F} \\
=0, & |\theta|<\theta_{F}
\end{array} ;\right. \\
& \epsilon^{-}(\theta)= \begin{cases}=0, & |\theta|>\theta_{F} \\
<0, & |\theta|<\theta_{F}\end{cases}
\end{aligned}
$$

Together $\epsilon^{ \pm}$make up a monotonic, smooth function via $\epsilon=\epsilon^{+}+\epsilon^{-}$. Comparing the two expressions for $\delta E$ and using the constraint on the variation in $\rho$ and $\tilde{\rho}$ coming from (74),

$$
\delta \rho+\delta \tilde{\rho}=\int d \theta^{\prime} \delta \rho\left(\theta^{\prime}\right) \Gamma_{++}\left(\theta-\theta^{\prime}\right)
$$

we arrive at equation (24).

\section{B Determination of Dressed Scattering Phase}

In this appendix we compute the dressed two body scattering phase of a particle-hole excitation. To compute this phase we examine how the momentum of one of the added excitations is altered by the addition of both excitations to the system.

The two-body scattering phase of the particle-hole excitation is defined by

$$
\delta_{p h}\left(\theta_{p}, \theta_{h}\right)=L\left(p_{p}\left(\theta_{p}\right)-p_{p 0}\left(\theta_{p}\right)\right),
$$

where $p_{p}(\theta)$ is the momentum of the particle when the particle/hole pair is present, while $p_{p 0}(\theta)$ is what the momentum of the particle would be without the hole and without the effect of the particle's presence on the sea of excitations already present in the $T=0$ ground state. 
Let $\theta_{p}, \theta_{h}$ be the rapidities of the particle and hole we intend to add to the system. Without having added either excitation, the dressed momentum of the particle is given by

$$
\begin{aligned}
p_{p 0}\left(\theta_{p}\right) & =\Delta \sinh \left(\theta_{p}\right)+2 \pi \int_{-\theta_{F}}^{\theta_{F}} d \theta n_{0}(\theta) \phi\left(\theta_{p}-\theta\right) ; \\
\phi(\theta) & =\frac{1}{2 \pi i} \log S_{++}(\theta),
\end{aligned}
$$

where $n_{0} \equiv \rho+\tilde{\rho}$ is the density of states given in (22). This is the density of states without either the particle or hole present. Once the particle and hole are added, the density of the ground-state sea is altered. Rather than being given by (22), it is governed by

$$
n(\theta)=\frac{\Delta}{2 \pi} \cosh (\theta)+\frac{1}{L}\left(\Gamma_{++}\left(\theta-\theta_{p}\right)-\delta\left(\theta-\theta_{h}\right)\right)+\int_{-\theta_{F}}^{\theta_{F}} d \theta^{\prime} n\left(\theta^{\prime}\right) \Gamma_{++}\left(\theta-\theta^{\prime}\right) .
$$

The $1 / L$ term $\left(\Gamma\left(\theta-\theta_{p}\right)-\delta\left(\theta-\theta_{h}\right)\right)$ represents the disturbance the particle-hole excitation produces in the density of states. The $\delta\left(\theta-\theta_{h}\right)$ arises from removing the particle to create the hole, while $\Gamma\left(\theta-\theta_{p}\right)$ arises from including the particle in the integral $\int d \theta \rho \Gamma_{++}$: the presence of this particle alters the density of states in the same way the continuum of particles in the ground state does.

Taking into account the alteration the addition the particle-hole excitation has upon the density of states, the momentum of the added particle becomes

$$
p_{p}\left(\theta_{p}\right)=\Delta \sinh \left(\theta_{p}\right)+2 \pi \int_{-\theta_{F}}^{\theta_{F}} d \theta n(\theta) \phi\left(\theta_{p}-\theta\right) .
$$

As $\theta_{F}$ is unshifted by the addition of the particle-hole pair we are able to write $n(\theta)$ as

$$
n(\theta)=n_{0}(\theta)+\frac{1}{L} n_{1}(\theta)-\frac{1}{L} \delta\left(\theta-\theta_{h}\right) .
$$

Substitution of (82) into (80) then allows the scattering phase to be reduced to

$$
\begin{aligned}
\delta_{p h}\left(\theta_{p}, \theta_{h}\right) & =-2 \pi \phi\left(\theta_{p}-\theta_{h}\right)+2 \pi \int_{-\theta_{F}}^{\theta_{F}} d \theta n_{1}(\theta) \phi\left(\theta_{p}-\theta\right) \\
n_{1}(\theta) & =\Gamma_{++}\left(\theta-\theta_{p}\right)-\Gamma_{++}\left(\theta-\theta_{h}\right)+\int_{-\theta_{F}}^{\theta_{F}} d \theta^{\prime} n_{1}\left(\theta^{\prime}\right) \Gamma_{++}\left(\theta-\theta^{\prime}\right) .
\end{aligned}
$$

We are interested in computing the scattering phase right at the Fermi surface, i.e. $\delta_{p h}\left(\theta_{p}=\right.$ $\left.\theta_{F}, \theta_{h}=\theta_{F}\right)$. But we then immediately see

$$
\delta_{p h}\left(\theta_{F}, \theta_{F}\right)=-2 \pi \phi(0)=-\pi
$$

Hence the low-energy particle-hole S-matrix is, $S=e^{i \delta_{p h}\left(\theta_{F}, \theta_{F}\right)}=-1$, as we claimed. Here we have focused upon the changes in the momentum of the particle. If we had examined the momentum of the hole instead we would have arrived at an identical conclusion. 


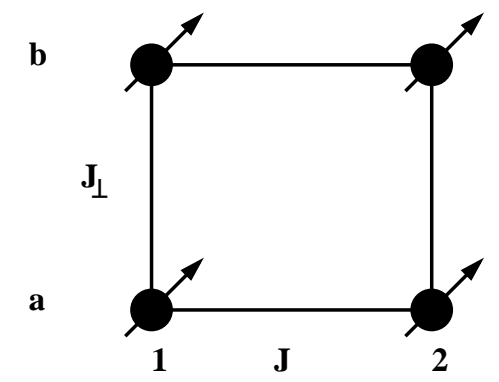

Figure 8: A two rung spin ladder.

\section{Reduction of a Ferromagnetic Spin-1/2 Ladder to an Effective Spin-1/2 Chain}

In this section we consider a map reducing a spin-1/2 ladder with ferromagnetic rung interactions in a magnetic field to an effective spin- $1 / 2$ chain. The essential idea behind the map is already discussed in a number of articles [30, 31]. However there the treatment considered a ladder with antiferromagnetic rung interactions. Here we adapt the map to ladders with ferromagnetic rungs.

For the sake of simplicity we consider a two-rung ladder, as pictured in Figure 8 . The Hamiltonian of the ladder is given by,

$$
\begin{aligned}
\mathcal{H}= & \mathcal{H}_{J}+\mathcal{H}_{J_{\perp}} \\
= & J\left(S_{a 1} \cdot S_{a 2}+S_{b 1} \cdot S_{b 2}\right) \\
& +J_{\perp \perp}\left(S_{a 1}^{+} S_{b 1}^{-}+S_{a 2}^{+} S_{b 2}^{-}+\text {h.c. }\right)+J_{\perp z}\left(S_{a 1}^{z} S_{b 1}^{z}+S_{a 2}^{z} S_{b 2}^{z}\right) \\
& +H\left(S_{a 1}^{z}+S_{b 1}^{z}+S_{a 2}^{z}+S_{b 2}^{z}\right) .
\end{aligned}
$$

We will suppose that $J>0$ while $J_{\perp \perp}<J_{\perp z}<0$.

We first treat the limit $J=0$ where the rungs decouple. The eigenstates on each rung with their corresponding energies are then

$$
\begin{aligned}
|+\rangle & =|\uparrow \uparrow\rangle ; \quad \frac{J_{\perp z}}{4}-H \\
|0\rangle & =\frac{1}{\sqrt{2}}(|\uparrow \downarrow\rangle+|\downarrow \uparrow\rangle) ; \quad \frac{J_{\perp \perp}}{2}-\frac{J_{\perp z}}{4} \\
|-\rangle & =|\downarrow \downarrow\rangle ; \quad \frac{J_{\perp z}}{4}+H \\
|s\rangle & =\frac{1}{\sqrt{2}}(|\uparrow \downarrow\rangle-|\downarrow \uparrow\rangle) ; \quad-\frac{J_{\perp \perp}}{2}-\frac{J_{\perp z}}{4},
\end{aligned}
$$

where the first three states are that of the triplet while the remaining state correspond to the singlet. The two states $|+\rangle$ and $|0\rangle$ are of lowest energy. It is these two states that we will use in constructing the effective spin- $1 / 2$ chain. 
To take into account $\mathcal{H}_{J}$ we perform second order perturbation theory. We find that the energies of the four basis states describing the low energy Hilbert space of both rungs, $|++\rangle$, $1 / \sqrt{2}(|+0\rangle \pm|0+\rangle)$, and $|00\rangle$, are shifted as follows:

$$
\begin{aligned}
|++\rangle: & \frac{J}{2} \\
1 / \sqrt{2}(|+0\rangle \pm|0+\rangle): & \pm \frac{J}{2} \\
|00\rangle: & \alpha \equiv \frac{J^{2}}{2\left(J_{\perp \perp}-J_{\perp z}\right)}+\frac{J^{2}}{8 J_{\perp \perp}} .
\end{aligned}
$$

Understanding $|+\rangle=|\tilde{\uparrow}\rangle$ and $|-\rangle=|\tilde{\downarrow}\rangle$, we can write down an effective system of interaction spin-1/2's:

$$
\mathcal{H}_{\text {eff }}=\frac{J_{\perp}^{e}}{2}\left(\sigma_{1}^{+} \sigma_{2}^{-}+\sigma_{2}^{+} \sigma_{1}^{-}\right)+J_{z}^{e} \sigma_{1}^{z} \sigma_{2}^{z}-H^{e}\left(\sigma_{1}^{z}+\sigma_{2}^{z}\right)+c,
$$

where the various effective couplings are given in terms of the original couplings by

$$
\begin{aligned}
J_{\perp}^{e} & =J \\
J_{z}^{e} & =J / 2+\alpha ; \\
H^{e} & =\frac{H}{2}+\frac{\alpha}{4}+\frac{1}{4}\left(J_{\perp \perp}-J_{\perp z}-\frac{J}{2}\right) ; \\
c & =\frac{J_{\perp \perp}}{2}-H+\frac{J}{8}+\frac{\alpha}{4} .
\end{aligned}
$$

We point out that as $\alpha$ can be negative, so can $J_{z}^{e} . J_{z}^{e}$ negative is essential to correctly describing the physics of the spin- 1 chain.

Having reduced the spin ladder with ferromagnetic rung couplings to a spin- $1 / 2$ chain we now bosonize. Applying the standard results (see [2]) we find that the associated Lagrangian is

$$
\begin{aligned}
\mathcal{L} & =\frac{K}{2 \pi} \partial^{\mu} \Phi \partial_{\mu} \Phi \\
K & =\frac{1}{\left(1+\frac{2 J_{z}^{e}}{\pi J^{e}}\right)},
\end{aligned}
$$

while the fields are given by

$$
\begin{aligned}
& n^{-}(x)=\frac{1}{\sqrt{2}}\left(S_{a}^{-}(x)+S_{b}^{-}(x)\right)=\sigma^{-}(x)=e^{-i \Phi(x)}\left(1+c_{1} \cos (2 \pi(\Theta(x)-M x))\right) \\
& M_{z}(x)=S_{a}^{z}(x)+S_{b}^{z}(x)=\sigma^{z}(x)+1 / 2=-\frac{K}{v_{F} \pi} \partial_{\tau} \Phi(x)+c \cos (2 \pi(\Theta(x)-M x)) .
\end{aligned}
$$

Here $M$ is the magnetization density of the spin- $1 / 2$ ladder (and so the corresponding spin- 1 chain). In terms of the magnetization, $M^{e}$, of the effective spin- $1 / 2$ chain, $M=M^{e}+1 / 2$. We see $n^{-}$is defined in terms of the $k_{y}=0$ component of the ladder spin operators. Here we thus 
expect $n^{-}$to encode power law correlations for wavevectors near $k=(\pi, 0)$. In a ladder with antiferromagnetic rungs, power law correlations would be seen instead for $k=(\pi, \pi)$.

With $J_{z}^{e}<0$ (provided $\alpha$ is sufficiently large) we see that $K>1$ in correspondence with both our analysis and the results of [33]. This marks out the main difference between ladders with ferromagnetic rungs and ladders with antiferromagnetic rungs. With antiferromagnetic rungs, a similar analysis leads to $J_{z}>0$ and so $K<1$ ([30, 31]). 


\section{References}

[1] F.D.M. Haldane, Phys. Lett. 93A (1983) 464.

[2] I. Affleck in Fields, Strings, and Critical Phenomena, eds. E. Brezin and J. Zinn-Justin, North-Holland (1990) Amsterdam.

[3] A. B. Zamolodchikov and Al. B. Zamolodchikov, Ann. of Phys. 120 (1979) 253.

[4] I. Affleck, T. Kennedy, E. Lieb, and H. Tasaki, Comm. Math. Phys 115 (1988) 477.

[5] H. M. Babujian, Phys. Lett. A 90 (1982) 479; ibid. Nucl. Phys. B 215 (1983) 317; P. Kulish, N. Reshetikhin, and E. Sklyanin, Lett. Math. Phys. 5 (1981) 393; L. Takhtajan, Phys. Lett. 87 A (1982) 479.

[6] J.B. Parkinson, J.C. Bonner, Phys. Rev. B 32 (1985) 4703; A. Moreo, Phys. Rev. B 35 (1987) 8562; T. Sakai and M. Takahashi, Phys. Rev. B 43 (1991) 13383; L. Campos Venuti, E. Ercolessi, G. Morandi, P. Pieri, and M. Roncaglia, cond-mat/9908044.

[7] H. Mutka, C. Payen, P. Molinié, J. L. Soubeyroux, P. Colombet, and A.D. Taylor, Phys. Rev. Lett. 67 (1991) 497.

[8] W.J.L. Buyers, R.M. Morra, R.L. Armstrong, M.J. Hogan, P. Gerlach, and K. Hirakawa, Phys. Rev. Lett. 56 (1986) 371; M. Steiner, K. Kakurai, J. K. Kjems, D. Petitgrand, and R. Pynn, J. Appl. Phys. 61 (1987) 3953.

[9] J.P. Renard, M. Verdaguer, L.P. Regnault, W.A.C. Erkelens, J. Rossat-Mignod, and W.G. Stirling, Europhys. Lett. 3 (1987) 945.

[10] E. Dagotto and T. M. Rice, Science 271 (1996) 618.

[11] M. Fabrizio, Phys. Rev. B 48 (1993) 15838; K. Kuroki and H. Aoki, Phys. Rev. Lett 72 (1994) 2947; H.J. Schulz, Phys. Rev. B 53 R (1996) 2959; L. Balents and M.P.A. Fisher, Phys. Rev. B 53 (1996) 12133; H.H. Lin, L. Balents, and M. Fisher, Phys. Rev. B 56 (1997) 6569; R. Noack, D. J. Scalapino, and S. White, Phil. Mag. B 74 (1996) 485; ibid., Physica C 270 (1996) 281; ibid., Europhys. Lett. 30 (1995) 163.; D. Duffy, S. Haas, and E. Kim cond-mat/9804221; H.H. Lin, L. Balents, M.P.A. Fisher, cond-mat/9801285.

[12] R.M. Konik, F. Lesage, A.W.W. Ludwig, H. Saleur, Phys. Rev. B 61 (2000) 4983; R.M. Konik and A.W.W. Ludwig, cond-mat/9810332

[13] H. J. Schulz, Phys. Rev. B 34 (1986) 6372.

[14] I. Affleck, Phys. Rev. B 41 (1990) 6697.

[15] A. B. Zamolodchikov and Al. B. Zamolodchikov, Nucl. Phys. B 379 (1992) 602.

[16] I. Affleck and R. Weston, Phys. Rev. B 45 (1992) 4667;

[17] E. Sorensen and I. Affleck, Phys. Rev B 49 (1994) 13235. 
[18] J. Sagi and I. Affleck, Phys. Rev. B. 53 (1996) 9188, cond-mat/9506137.

[19] R. M. Konik, Haldane Gapped Spin Chains: Exact Low Temperature Expansions of Correlation Functions, cond-mat/0105284.

[20] S. Sachdev and K. Damle, Phys. Rev. Lett 78 (1997) 943, cond-mat/9610115.

[21] K. Damle and S. Sachdev, Phys. Rev. B 57 (1998) 8307, cond-mat/9711014.

[22] D. W. Jepsen, J. Math. Phys. 6 (1965) 405.

[23] F.D.M. Haldane, J. Phys. A 15 (1982) 507.

[24] E. Papa and A. M. Tsvelik, cond-mat/0006422.

[25] F. Lesage and H. Saleur, Nucl. Phys. B 490 (1997) 543, cond-mat/9612050, F. Lesage and H. Saleur, Nucl. Phys. B 493 (1997) 613, cond-mat/9611025.

[26] I. Affleck, Phys. Rev. B 43 (1991) 3215.

[27] F.D.M. Haldane, Phys. Rev. Lett, 47 (1981) 1840.

[28] D. Loss and B. Normand, cond-mat/9804151; B. Normand, J. Kyriakidis, and D. Loss, cond-mat/9902104; G. Fáth and P. Littlewood, cond-mat/9810259.

[29] S. Sachdev and K. Damle, J. Phys. Soc. of Japan 69 (200) 2712.

[30] F. Mila, cond-mat/9805029 K. Totsuka, Phys. Rev. B 57 (1998) 3454.

[31] T. Giamarchi and A. Tsvelik Phys. Rev. B 59 (1999) 11398.

[32] R. Chitra and T. Giamarchi, Phys. Rev. B 55 (1997) 5816.

[33] D. Shelton, A. A. Nersesyan, and A. Tsvelik, Phys. Rev. B 53 (1996) 8521; A. Furusaki and S.C. Zhang, Phys. Rev. B 60 (1999) 1175;

[34] M. Takahashi and T. Sakai, J. Phys. Soc. of Japan, 60 (1991) 760.

[35] V. Korepin, N. Bogoliubov, and A. Izergin, Quantum Inverse Scattering Method and Correlation Functions, Cambridge University Press (1993) Cambridge.

[36] Z. Honda, K. Katsumata, H. Aruga Katori, K. Yamada, T. Ohishi, T. Manabe and M. Yamashita, J. Phys.: Condensed Matter 9 (1997) L83; ibid. 3487; Z. Honda, H. Asakawa and K. Katsumata, Phys. Rev. Lett. 81 (1998) 2566.

[37] Y. Ajiro, T. Goto, H. Kikuchi, T. Sakakibara, and T. Inami, Phys. Rev. Lett 63 (1989) 1424.

[38] P. Hasenfratz, M. Maggiore and F. Niedermayer, Phys. Lett. B245 (1990) 522

[39] P. Wiegmann, JETP Lett. 41 (1985) 95; A. Tsvelik, Sov. Phys. JETP 66 (1987) 221. 
[40] P. Fendley, Integrable perturbed coset models and sigma models, hep-th/0101034.

[41] D. Orgad, cond-mat/0005181.

[42] Y. Imry, Phys. Rev. B 13 (1976) 3018.

[43] H.J. Schulz and C. Bourbonnais, Phys. Rev. B 27 (1983) 5856; A. Luther and I. Peschel, Phys. Rev. B 9 (1974) 2911.

[44] A. Zheludev, Y. Chen, C. Broholm, Z. Honda, and K. Katsumata, cond-mat/0003223

[45] O. Golinelli, T. Jolicoeur, and R. Lacaze, J. Phys. Condens. Matter 5 (1993) 7847; S. Meshkov, Phys. Rev. B 48 (1993) 6167.

[46] N. Nagaosa and S. Murakami, J. Phys. Soc. Japan 67 (1998) 1876. 